\title{
Multi-carrier modulation analysis and WCP-COQAM proposal
}

\author{
Hao Lin* and Pierre Siohan
}

\begin{abstract}
In the vision towards future radio systems, where access to information and sharing of data is to be available anywhere and anytime to anyone for anything, a wide variety of applications and services are therefore envisioned. This naturally calls for a more flexible system to support. Moreover, the demand for drastically increased data traffic, as well as the fact of spectrum scarcity, would eventually force future spectrum access to a more dynamic fashion. For addressing the challenges, a powerful and flexible physical layer technology must be prepared, which naturally brings us to the question whether the legacy of the OFDM system can still fit in this context. In fact, during the past years, extensive research effort has been made in this area and several enhanced alternatives have been reported in the literature. Nevertheless, up to date, all of the proposed schemes have advantages and disadvantages. In this paper, we give a detailed analysis on these well-known schemes from different aspects and point out their open issues. Then, we propose a new scheme that aims to maximally overcome the identified drawbacks of its predecessors while still trying to keep their advantages. Simulation results illustrate the improvement achieved by our proposal.
\end{abstract}

Keywords: FBMC; FMT; GFDM; OFDM; OQAM

\section{Introduction}

In today's mobile communication systems, cyclic-prefixbased orthogonal frequency division multiplexing (CPOFDM) is widely adopted. It shows that the concept of multi-carrier modulation (MCM) is well recognized as an efficient mode for broadband transmission. However, for moving towards future radio systems beyond 2020, it is time to ask whether the traditional fashion for MCM can effectively meet particular demands from some emerging scenarios, e.g., ultra dense networks (UDN) and heterogenous networks (HetNet). To be more specific, future radio systems aim to offer drastically increased data volume. To address this challenge, an envisioned approach is to increase the network densification, together with dynamic spectrum access (DSA) techniques based on the cognitive radio (CR) concept, leading to the so-called UDN [1]. In order to effectively implement DSA solutions, it relies on the physical layer modulations. Indeed, an important requirement is that the radio signal must provide a good spectrum shape, i.e., low out-of-band radiation. However, an identified drawback of the OFDM systems is that the

*Correspondence: hao.lin@orange.com

Orange Labs, 4 Rue du Clos Courtel, Cesson-Sévigné 35512, France employed rectangular pulse yields an unsatisfactory sidelobe attenuation which may cause severe consequences in UDN. For instance, the high out-of-band radiation may severely pollute the neighbors in the adjacent bands. The second drawback of OFDM is the lack of waveform flexibility which could be problematic in the HetNet. Considering the heterogeneity of future networks, where cells with different sizes are integrated, doubly dispersive, i.e., time/frequency-dispersive, channel appears in the signal transmission environment, while the dominant parts can be varying from time to time [2]. Research analysis has shown that the waveform localization property in time and frequency domains both play an important role in the design criteria for addressing the two-dimensional fading [3]. Moreover, in a system with imperfect synchronization conditions, a waveform that is designed with different weights towards time and frequency localization will provide different degree of robustness against the time and frequency residual synchronization errors as well $[4,5]$. Therefore, a suitable system should be able to employ flexible waveforms, depending on different transmission circumstances in future HetNet scenarios.

To overcome these two drawbacks, during the past decades, people tried to improve the OFDM with some

\section{Springer}

(C) 2014 Lin and Siohan; licensee Springer. This is an Open Access article distributed under the terms of the Creative Commons Attribution License (http://creativecommons.org/licenses/by/2.0), which permits unrestricted use, distribution, and reproduction in any medium, provided the original work is properly credited. 
other pulse shapes. However, the mathematicians proved that for a MCM system it cannot simultaneously employ a flexible pulse, remain orthogonality, and transmit at the Nyquist rate. This statement was later known as the Balian-Low Theorem (BLT) [6]. Thus, novel MCM transmission fashion should be used to solve this bottleneck. In the literature, two main MCM alternatives have been proposed. The first scheme is called Filter Bank Multi-carrier/Offset Quadrature Amplitude Modulation (FBMC/OQAM), whose pioneering work of Saltzberg [7] can be also analyzed in the Gabor perspective. A key idea of his multi-carrier system is to split the complex data symbols into their real and imaginary parts. By this way, the orthogonality condition is relaxed and only applies to the real field, which eventually allows us to escape from the requirements defined by the BLT. The resulting degree of freedom can then be used to improve the signal spectrum shape and further to address the waveform flexibility. On the other hand, the second scheme, which is called Filter Multi-Tone (FMT), consists in relaxing the Nyquist rate transmission by employing an over Nyquist sampling, which, in practice for a given symbol duration, leads to an increased frequency spacing. This means that if the pulse spectrum is band-limited to the extended subcarrier spacing, no interference exists between the subcarriers. Therefore, the waveform design only needs to satisfy a one-dimensional Nyquist condition, e.g., square root raised cosine (SRRC), leaving more room for the signal spectrum improvement as well as the waveform flexibility. The first proposal in this direction dates back from 1971 [8] and it was later known under different acronyms, such as fraction-spaced multi-carrier modulation [9], over-sampled OFDM [10], over-sampled DFT [11], and more recently, it is often denoted as FMT [12]. Aside from the above-mentioned advantages, both FBMC/OQAM and FMT have some weak points. Since there is no CP in the FBMC/OQAM and FMT, the system orthogonality is ruined when the transmission passes through a multi-path channel. Therefore, more equalizers with higher complexity are needed on the receiver side for maintaining an acceptable interference level [13-15]. Moreover, due to this orthogonality loss, even more complex receiver is requested when further considering these schemes in combination with space-time encoded multiple-input-single-output (MISO) transmission, e.g., Alamouti coding [16]. In addition, for the FMT scheme, due to its over-sampled nature, it inherits a spectral efficiency (SE) loss.

Besides these two schemes, a new concept of MCM has recently appeared in the literature. It replaces the linear filtering with a circular filtering for pulse shaping. This idea was originally raised with the introduction of generalized frequency division multiplexing (GFDM) [17]. By using a circular filtering, the overall MCM system can maintain a block transform processing so that a $\mathrm{CP}$ can be easily inserted. Moreover, an SRRC pulse is used in the GFDM scheme to further improve the signal power spectrum density (PSD). Due to this advantage, the GFDM was first presented for communications over TV white space. Later, its good out-of-band energy attenuation rapidly captured a lot of attention in the CR field [18-21] and further towards next generation of cellular systems [22]. However, a primary obstacle is that the GFDM is not an orthogonal system, again, restricted by the BLT [23]. This non-orthogonality nature could be a vital drawback in a practical usage. The in-band interference severely causes a performance degradation, which further was proved to be pulse shape dependent [23]. To mitigate the performance degradation, one possible solution is to use an iterative interference cancelation [20]. However, the receiver complexity gets largely increased and might even get exploded when considering it together with MIMO transmission. After GFDM, the circular filtering concept is further adopted for the FMT, which gives the birth for the cyclic block FMT (CB-FMT) [24]. It can successfully overcome the orthogonality issue. Nevertheless, as the modulation kernel remains FMT, the SE loss as well as the trade-offs between orthogonality and waveform flexibility still exist.

To the best of our knowledge, a comparative analysis among all of the above-mentioned MCM schemes has never been reported in the literature. In this paper, we first provide such analysis among these MCM schemes following four main criteria: spectral efficiency, power spectral density, orthogonality for distortion-free/multi-path channel, and waveform flexibility. Although the selected four criteria do not fully cover all of the research aspects, they can already be used to identify some drawbacks of each of these schemes. Then, motivated by the analysis, we further introduce a new type of FBMC/OQAM, which also adopts the circular filtering to the FBMC/OQAM and we call it FBMC/circular OQAM (COQAM) a The investigation of this new scheme aims to overcome the identified drawbacks of the State-of-the-Art (SoTA) MCM schemes. The rest of the paper is organized as follows: in Section 2, we give a brief recall of the SoTA MCM schemes and provide an analysis on their pros and cons with regard to four criteria. In Section 3, we present the concept of the FBMC/COQAM modulation and its motivation behind. In Section 4, the efficient implementation algorithm is presented. In Section 5, we illustrate a detailed transceiver design by considering the FBMC/COQAM in a multipath transmission context. The efficiency evaluations of the proposed scheme are given in Section 6. In Section 7, some discussions and remarks are addressed to further clarify the proposal interest. Finally, in Section 8, we draw some conclusions and perspectives. For simplicity, in the following, we omit the term FBMC for FBMC/OQAM and FBMC/COQAM. 


\section{SoTA MCM schemes analysis}

In this section, we first give a brief description of the SoTA schemes and their mathematical expressions. Then we provide an analysis on the pros and cons from different aspects, which will motivate our research proposal.

\subsection{SoTA MCM description}

In what follows, the five MCM schemes, i.e., CP-OFDM, OQAM, FMT, GFDM, and CB-FMT, are briefly recalled, including their general concepts and baseband expressions.

\subsubsection{CP-OFDM modulation}

OFDM is the simplest MCM system and it is a widely adopted in many applications. Different from the singlecarrier modulation for which the transmitted data is spread over a wide bandwidth, in OFDM the data are modulated at a set of narrow subcarriers whose bandwidth is largely smaller than the channel coherent bandwidth, leading to quasi flat fading at each subcarrier. Moreover, a CP is inserted in front of each OFDM symbol, which is the copy of the tail samples. This further ensures a true flat fading at each subcarrier, therefore, enhancing the robustness against frequency selective fading. The baseband OFDM symbol, without CP insertion, can be expressed as

$$
s_{\mathrm{OFDM}}[k]=\sum_{m=0}^{M-1} c_{m} e^{\frac{j 2 \pi m k}{M}}
$$

with $M$ the number of subcarriers and $c_{m}$ the complexvalued data symbols, e.g., QAM constellations. The overall CP-OFDM system can be efficiently realized by fast Fourier transform (FFT/IFFTs), and one important advantage is that the CP-OFDM can maintain a full orthogonality, which requires only a simple equalizer at the receiver. However, it employs a rectangular pulse with several disadvantages that will be analyzed later on. It is worth noting that since the rectangular pulse is used in OFDM system, the pulse shaping is implicitly realized by the Fourier transform.

\subsubsection{OQAM modulation}

The remarkable contribution of the OQAM concept is that it introduced a staggered transmission structure, which allows it to escape from the BLT [25]. Hence, the OQAM scheme can simultaneously employ an improved pulse shape, keep full orthogonality, and transmit at the Nyquist rate. Contrary to the OFDM scheme that transmits complex-valued at subcarriers, in the staggered structure, the real and imaginary parts of the complexvalued symbols are transmitted separately with a delay of half OFDM symbol duration. More details for the OQAM concept can be found in [26] and the references therein. The baseband OQAM-modulated signal writes as [27]

$$
s_{\mathrm{OQAM}}[k]=\sum_{m=0}^{M-1} \sum_{n \in \mathcal{Z}} a_{m, n} \underbrace{g\left[k-n N_{1}\right] e^{j \frac{2 \pi}{M} m\left(k-\frac{D}{2}\right)} e^{j \phi_{m, n}}}_{g_{m, n}[k]},
$$

where $g$ is the prototype filter with a length of $L_{g}$ and $D=L_{g}-1$ (here, $g$ is assumed to be real-valued and symmetrical); $N_{1}=M / 2$ is the discrete-time offset; $\phi_{m, n}$ is an additional phase term at subcarrier $m$ and symbol index $n$ which can be expressed as $\frac{\pi}{2}(n+m)$. The transmitted symbols $a_{m, n}$ are real-valued, which can be obtained from a QAM constellation by taking the real and imaginary parts. To address a perfect reconstruction of real symbols, the prototype filter must satisfy the orthogonality condition:

$$
\Re\left\{\sum_{k \in \mathcal{Z}} g_{m, n}[k] g_{p, q}^{*}[k]\right\}=\delta_{m, p} \delta_{n, q}
$$

where * denotes the complex conjugation, $\delta_{m, p}=1$ if $m=$ $p$ and $\delta_{m, p}=0$ if $m \neq p$. The OQAM system only holds a perfect orthogonality in the distortion-free case.

\subsubsection{FMT modulation}

The FMT concept was originated from the Filter Bank (FB) theory [28]. It showed that in a critically sampled FB system, only rectangular prototype filter can hold a perfect reconstruction. This is an alternative interpretation of BLT. Therefore, the FMT shifts to a non-critical sampled system by introducing an over-sampling at each subcarrier so that the additional degree of freedom can be traded for some better designed prototype filters [12]. The baseband FMT signal is written as

$$
s_{\mathrm{FMT}}[k]=\sum_{m=0}^{M-1} \sum_{n \in \mathcal{Z}} c_{m, n} \underbrace{h\left[k-n N_{2}\right] e^{j \frac{2 \pi k m}{M}}}_{h_{m, n}[\mathrm{k}]},
$$

with $M$ the number of subcarriers, $c_{m, n}$ the complexvalued data symbols, and $N_{2}$ the over-sampling factor, i.e., $N_{2}>M$. To hold a perfect reconstruction, the prototype filter $h$ must meet

$$
\sum_{k \in \mathcal{Z}} h_{m, n}[k] h_{p, q}^{*}[k]=\delta_{m, p} \delta_{n, q} .
$$

If the employed pulse has a spectrum that is restrictively limited within the increased subcarrier spacing, no spectra-crossing happens between adjacent subcarriers, which confirms an inter-carrier interference (ICI) free transmission. Thus, the orthogonality condition (5) is changed to a one-dimension only, i.e., any traditional Nyquist pulse can be used. This can be interpreted as an alternative way to relax the orthogonality condition. Moreover, like OQAM, the FMT only holds a perfect reconstruction in the distortion-free case as well. 


\subsubsection{GFDM modulation}

The idea of GFDM is to group a set of complex-valued symbols from time-frequency lattice into one block. Then, for each block, a subcarrier-wise processing is carried out, which includes the up-sampling, pulse shaping, and tail biting, and finally is followed by a modulation operation to a set of subcarrier frequencies ( $c f$. [17], Fig. One). The baseband GFDM-modulated signal of one block, i.e., for $k \in[0, M K-1]$, is expressed as

$$
s_{\mathrm{GFDM}}[k]=\sum_{m=0}^{M-1} \sum_{n=0}^{K-1} c_{m, n} \tilde{f}[k-n M] e^{\frac{j 2 \pi k m}{N}},
$$

with $M$ as the subcarrier number and $K$ as the number of symbol slots considered in one block. The pulse shape $\tilde{f}[k]$ indicates a periodic repetition of the prototype filter $f[k]$ with a period of $M K$, i.e.,

$$
\tilde{f}[k]=f[\bmod (k, M K)] .
$$

The periodic filter is used to realize the circular convolution at the transmitter, which is equivalent to the tail-biting process [17]. Note that there does not exist any orthogonality condition for the filter design because the GFDM itself is a non-orthogonal system.

\subsubsection{CB-FMT modulation}

The CB-FMT scheme adopts the circular filtering on the top of traditional FMT modulation. By this change, the FMT can turn to a block transform processing such that a CP can be easily appended in front of each block, which therefore enhances the robustness against the multi-path fading. The baseband expression of CB-FMT is similar to (4) only with a circular filtering instead [24], i.e.,

$$
s_{\mathrm{CB}-\mathrm{FMT}}[k]=\sum_{m=0}^{M-1} \sum_{n=0}^{K-1} c_{m, n} \tilde{h}\left[k-n N_{2}\right] e^{j \frac{2 \pi k m}{M}},
$$

with

$$
\tilde{h}[k]=h\left[\bmod \left(k, N_{2} K\right)\right] .
$$

Note that if we set $N_{2}=M$, then the CB-FMT turns to be GFDM. Thus, the best filter that can guarantee (5) is only rectangular filter.

\subsection{Pros and cons analysis}

In this section, we provide a detailed analysis on the advantages and drawbacks of the above schemes. This analysis is conducted from four aspects, namely SE, PSD, orthogonality, and waveform flexibility.

\subsubsection{SE analysis}

For a multi-carrier system, denoting $F_{0}$ as the spacing between subcarriers and $T_{0}$ as the symbol duration, the modulated signal can be written as a linear combination of a Gabor family, reflecting a lattice form time-frequency representation. The maximum SE, for an orthogonal system, is reached when the symbol duration and the subcarrier spacing satisfy that

$$
T_{0} \cdot F_{0}=1 \text {. }
$$

Indeed, the measure of SE is inversely proportional to the product (8). If the product value gets greater, it means that there exists a SE loss in either time domain, i.e., taking longer time to transmit one symbol or in frequency domain, i.e., using more frequency bands for the transmission or the combination of both causes. Denoting by SEI, the spectral efficiency indicator, we have $0 \leq \mathrm{SEI} \leq 1$ with $\mathrm{SEI}=1$ being the optimum.

The CP-OFDM cannot achieve this maximum value due to the addition of a CP of length $T_{\mathrm{CP}}$ [29]. This leads to an overall efficiency reduction with the following:

$$
\mathrm{SEI}_{\mathrm{CP}-\mathrm{OFDM}}=\frac{1}{\left(T_{0}+T_{\mathrm{CP}}\right) \cdot F_{0}}=\frac{T_{0}}{T_{0}+T_{\mathrm{CP}}}<1 .
$$

On the contrary, the OQAM scheme respects the Nyquist rate and no CP is used. Hence, it is able to achieve the maximum efficiency [26], i.e.,

$$
\mathrm{SEI}_{\mathrm{OQAM}}=\frac{1}{T_{0} \cdot F_{0}}=1
$$

Although the FMT also does not use any CP, it cannot achieve maximum spectral efficiency due to its increased subcarrier spacing. In a FMT realization, this is generally embodied in the addition of a frequency domain roll-off factor $\alpha=\left(N_{2}-M\right) / M$, yielding the time-frequency efficiency [12]:

$$
\mathrm{SEI}_{\mathrm{FMT}}=\frac{1}{T_{0} \cdot F_{0}^{\prime}}=\frac{T_{0}}{(1+\alpha) \cdot T_{0}}<1 .
$$

For the GFDM modulation, since it uses the same CP length as in the CP-OFDM modulation, they normally address the similar time-frequency efficiency. Nevertheless, it is possible for the GFDM to group $K$-modulated symbols to one block with one CP appending ahead [17]. One may understand it in the way that the inserted CP is shared by $K$-modulated symbols, which is one of the main differences between CP-OFDM and GFDM in terms of spectral efficiency, leading the time-frequency efficiency of GFDM to

$$
\mathrm{SEI}_{\mathrm{GFDM}}=\frac{1}{\left(T_{0}+T_{\mathrm{CP}} / K\right) \cdot F_{0}}=\frac{T_{0}}{T_{0}+\frac{T_{\mathrm{CP}}}{K}}<1 .
$$

In a special case where $K=1$, its SEI is identical to that of CP-OFDM.

Likewise, the CB-FMT scheme also employs a CP with the same length as those of GFDM and CP-OFDM. Moreover, as it keeps the FMT kernel, the subcarrier spacing is also extended due to the over-sampled nature. This 
results the CB-FMT in a two-dimensional SE loss [24]. The time-frequency efficiency of CB-FMT system yields

$$
\mathrm{SEI}_{\mathrm{CB}-\mathrm{FMT}}=\frac{1}{\left(T_{0}+T_{\mathrm{CP}} / K\right) \cdot F_{0}^{\prime}}=\frac{T_{0}}{\left(T_{0}+\frac{T_{\mathrm{CP}}}{K}\right)(1+\alpha)}<1 .
$$

\subsubsection{PSD analysis}

As stated in Section 1, the radio signal PSD may become even more important in future radio systems. Due to the spectrum scarcity, the physical layer modulation must provide an improved radio signal PSD for enabling envisioned DSA techniques. In fact, all the SoTA alternatives have claimed that they have better signal PSD than the CP-OFDM systems. In Figure 1, we show the estimated PSD corresponding to the SoTA schemes. Our estimation is based on Welch's method ${ }^{\mathrm{b}}$ [30]. To ensure a fair comparison, the same prototype filter, which is the SRRC filter, ${ }^{\mathrm{C}}$ is used for these schemes except for CP-OFDM. The comparison result clearly shows that all the alternative schemes to CP-OFDM provide enhanced PSD shape with greatly reduced out-of-band radiations. A further relative comparison among them reveals that the OQAM and FMT schemes can outperform the others. This is because the OQAM and FMT are not block-transform-based processing. Therefore, there does not exist any spectral leakage due to the waveform discontinuity [31]. However, such spectral leakage still exists for the GFDM and CB-FMT systems which are realized with the block transform processing, resulting in an increased out-of-band radiation. Moreover, it can be clearly seen from the estimated PSD that keeping the same number of active subcarriers for the FMT and CB-FMT leads to a larger effective bandwidth

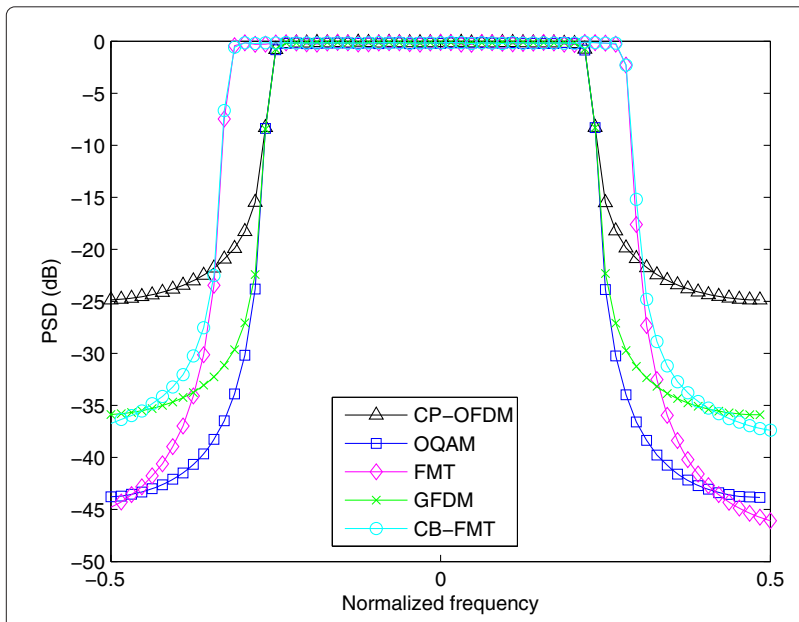

Figure 1 Estimated PSD corresponding to the SoTA schemes. PSD evaluation: $M=64$ and $M / 2$ active carriers; $C P$ length $M / 4$ for CP-OFDM, GFDM, and CB-FMT; SRRC filter of roll-off factor 0.2 with overlapping factor 32 for OQAM, FMT, GFDM, and CB-FMT;

$N_{2}=(5 / 4) M$ for FMT and CB-FMT; $K=32$ for GFDM and CB-FMT due to their increased subcarrier spacing. Thus, under a condition of keeping the same effective bandwidth, the FMT and CB-FMT must leave more carriers inactive.

\subsubsection{Orthogonality analysis}

The orthogonality is analyzed in two cases, i.e., end-toend (E2E) distortion-free case and multi-path (MP) channel case. The former shows whether the MCM can form a perfect reconstruction system. While the latter reveals the robustness against a frequency selective fading, meaning whether a simple one-tap equalizer can completely remove the multi-path interference, as such in the $\mathrm{CP}$ OFDM case. It hints further on the complexity of the receiver design. This feature is particularly important for the case of CP-OFDM-based space-time encoded MISO transmission, since the space-time decoding can therefore be simply realized by a one-tap equalization, resulting in a low-complexity receiver. Nevertheless, there still remain open issues when combining space-time encoded MISO with some MCM schemes other than CP-OFDM. A well-known example is the possibility of enabling a lowcomplexity Alamouti transmission with advanced MCM schemes [16]. Thus, in general, more weights are given to the orthogonality than to the SE for the MCM evaluation. In the following, we assume that the inserted CP is always sufficiently longer than the MP channel length for CP-OFDM, GFDM, and CB-FMT. Note that the Doppler effect is not considered in our orthogonality analysis, as it ruins the orthogonality for all the MCM schemes in general. The robustness against Doppler effect will be investigated in future publications.

The CP-OFDM system is a well-known orthogonal system. It holds the perfect reconstruction in the E2E case. In a MP case, with the aid of CP, neither the ICI nor the inter-symbol interference (ISI) is resulted. Hence, the orthogonality is fully addressed in CP-OFDM systems. Thanks to this advantage, the CP-OFDM is recognized as a low-complexity system because the channel compensation can be easily accomplished with a simple one-tap equalization ${ }^{\mathrm{d}}$.

For the rest four MCM schemes, we use error vector magnitude (EVM) to evaluate their orthogonality performance. The EVM, sometimes also called constellation error magnitude, is defined as

$$
\operatorname{EVM}(\mathrm{dB})=10 \log _{10}\left(\frac{\sum_{m, n}\left|c_{m, n}-\hat{c}_{m, n}\right|^{2}}{\sum_{m, n}\left|c_{m, n}\right|^{2}}\right)
$$

with $c_{m, n}$ the transmitted data constellations and $\hat{c}_{m, n}$ the received data constellations without hard decision. Note that in an E2E case, the received data constellations are at the demodulator output, while in the MP case, they are placed at the output of the equalization, which in this paper is a one-tap zero-forcing (ZF). The MP channel is 
simple two-path static channel with the second path of half channel gain of the first path.

Again, the SRRC prototype filter is used for OQAM, FMT, GFDM, and CB-FMT. The detailed parameters are given in Figure 2, where the orthogonality performances are displayed as a function of the filter roll-off (RO) factor. This reflects the orthogonality influence with regard to different time-frequency localization weights of the prototype filter. Further, no background noise is assumed to illustrate a pure impact on the constellation error due to the non-orthogonality.

The evaluation result shows that the OQAM system is nearly orthogonal in the E2E case, since the curve remains under a low EVM value, except for small RO factors close to zero. This can be explained by the uncertainty principle ([28], Ch. 11), i.e., if a window is narrow in time domain, it is broad in frequency domain and vice versa. The SRRC pulse with small $\mathrm{RO}$ factor tends to a sinc pulse which holds the orthogonality only when the pulse duration goes to infinity. This also explains why the EVM of OQAM decreases as the RO factor increases. It is because the SRRC pulse with higher RO factor has better time domain localization. Thus, considering a SRRC pulse with a finite duration, it holds more tightly the perfect reconstruction. On the other hand, both the FMT and CB-FMT scheme have similar orthogonality performance in the E2E case. This is not a surprising result as they share the same modulation kernel. Moreover, the result shows that they can maintain the orthogonality only if the RO factor does not exceed a certain threshold, whose value is calculated as

$$
1+r=\frac{N_{2}}{M} \Rightarrow r=\frac{N_{2}-M}{M} \text {. }
$$

Beyond this threshold, the spectra of the adjacent subcarriers start to reach across with each other, preventing

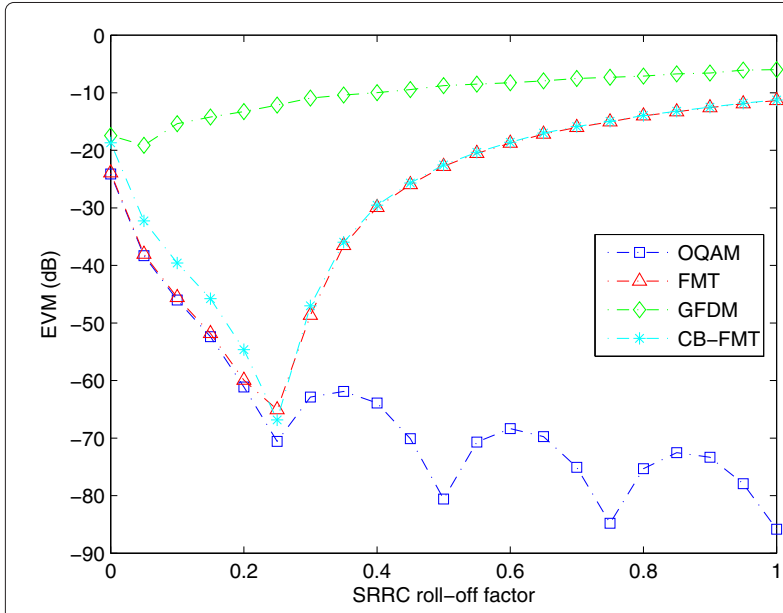

Figure 2 EVM evaluation E2E case, SRRC filter with overlapping factor $32, M=64, N_{2}=(5 / 4) M, K=32$ for GFDM and CB-FMT. All carriers are modulated. from the ICI-free assumption. Indeed, in line with [12], the wider the pulse spectrum is, the severer the ICI will be resulted. That is why the EVM curves for FMT and CB-FMT turn around at the threshold RO point and continuously go up as the RO factor increases. The GFDM scheme provides the worst orthogonality performance in the E2E case due to its non-orthogonal nature.

Moving to the MP case, as shown in Figure 3, the orthogonality for the OQAM and FMT is severely ruined due to the lack of CP. This confirms their drawback that the OQAM and FMT can only hold the orthogonal system in the distortion-free case. Therefore, in practice, either multi-tap equalizers [32,33] or multi-step equalizers $[13,14]$ must be considered for OQAM and FMT $[12,15]$ to mitigate the performance degradation. Similar to the E2E case, the GFDM continues badly performing due to its orthogonality loss. This degradation cannot be relieved with the aid of CP. For this reason, the GFDM receiver has to employ complex interference cancelation techniques, e.g., [34]. On the contrary, the CB-FMT keeps the same performance as in the E2E case. It reveals that the MP channel does not impact the orthogonality feature of the CB-FMT, and it further points out that the only impacting factor for the orthogonality of CB-FMT is the time-frequency localization property of the prototype filter which brings to our next analysis item: waveform flexibility.

\subsubsection{Waveform flexibility analysis}

In this paper, the waveform flexibility is evaluated under two conditions: (1) the capability for MCM schemes to employ a non-rectangular filter without any penalty on orthogonality; (2) the capability for MCM schemes to employ a filter, which can be localized either in frequency

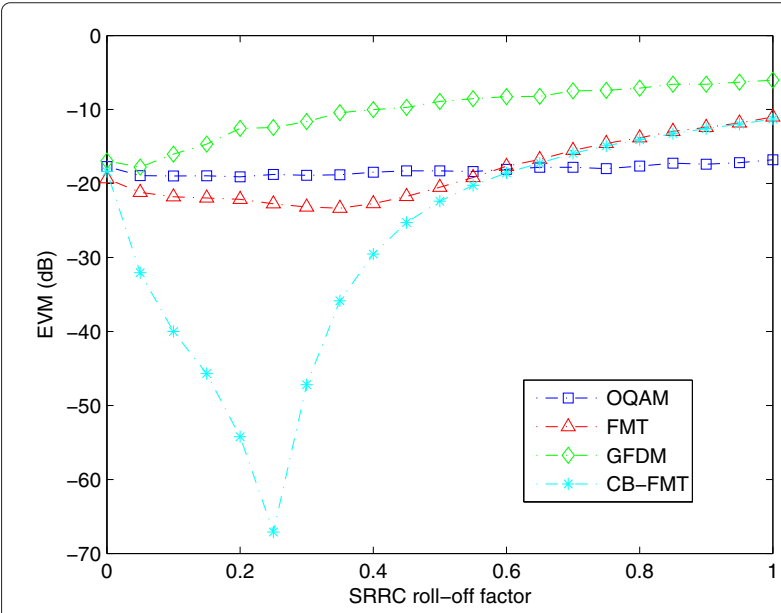

Figure 3 EVM evaluation MP case and two-path channel of length $\boldsymbol{M} / \mathbf{8}$. The second path has $\mathbf{5 0} \%$ channel gain of the first path. SRRC filter with overlapping factor $32, M=64, N_{2}=(5 / 4) M$, and $K=32$ for GFDM and CB-FMT. 
or in time domain, without any penalty. The evaluation on the first condition is straightforward. But for the second condition, we analyze the orthogonality degradation by varying the $\mathrm{RO}$ factor of the filter, which reflects the penalty by employing the filters with different time-frequency localization combinations. The CPOFDM uses a fixed rectangular pulse. Hence, it does not have any degree of flexibility. Unlike CP-OFDM, the OQAM scheme can use a prototype filter having either good time localization or good frequency localization or a good compromise. This is confirmed by Figure 2 that its perfect reconstruction is not significantly impacted as the RO factor changes. While for the FMT and CB-FMT, the orthogonality analysis reveals that well frequencylocalized pulses are preferred. This remark is actually in line with the statement given in ([24], Sec.V.B). On the other hand, one could improve the waveform flexibility of FMT and CB-FMT by increasing the over-sampling factor $N_{2}$. In line with (10), in Figure 4, it is shown that as $\mathrm{N}_{2}$ increases the RO factor threshold for which the FMTbased schemes can maintain orthogonality shifts to the right side. This indeed can be interpreted that more waveform flexibility can be achieved for CB-FMT but at the price of more SE loss. Finally, the GFDM scheme is a special case. Since it cannot hold a perfect reconstruction condition, the waveform flexibility does not make much sense in this context.

\section{COQAM: motivation and concept}

As our analysis showed, the CP-OFDM cannot address any flexibility and its PSD has a high out-of-band radiation. The drawbacks of OQAM and FMT are lack of the orthogonality under multi-path channel, which requires more complicated receiver design and limits the

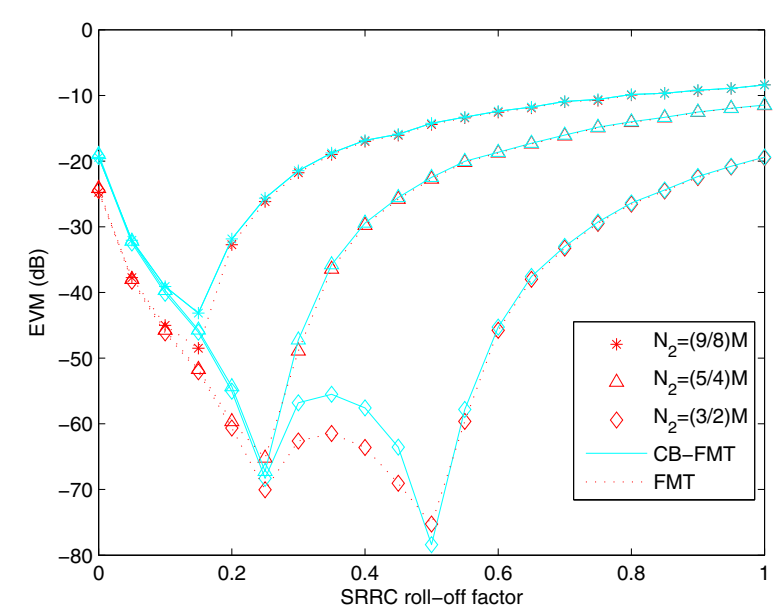

Figure 4 EVM evaluation E2E case. SRRC filter of overlap factor 32, $M=64, N_{2}=\{9 / 8,5 / 4,3 / 2\} M, K=32$ for CB-FMT. feasibility with MIMO transmission. Even more severe orthogonality issue is inherited in the GFDM scheme, which further increases the system complexity and prevents waveform flexibility. The CB-FMT can completely solve the orthogonality issue under some constraints on the pulse design and it manages to improve the PSD shape compared with CP-OFDM. However, a compromise is laid among waveform flexibility, orthogonality, and SE loss. In addition, the CB-FMT inherits a two-dimensional SE loss.

Knowing the shortcomings, an intuitive question is whether we can find an improved modulation that keeps most of the benefits from the predecessors and gets rid of their drawbacks. With this motivation, we investigated a new MCM scheme called COQAM, whose idea is to replace the linear convolution used in the OQAM with a circular convolution, similar to the GFDM and CB-FMT. By this way, we get a modulation scheme that corresponds to a block transform. So that a CP can be easily added to enhance the orthogonality. Partly derived from (2), the discrete-time COQAM signal $s[k]$ defined in a block interval such that $k \in\left[0, M K^{\prime}-1\right]$ is expressed as

$$
s_{\mathrm{COQAM}}[k]=\sum_{m=0}^{M-1} \sum_{n=0}^{K^{\prime}-1} a_{m}[n] \tilde{g}\left[k-n N_{1}\right] e^{j \frac{2 \pi}{M} m\left(k-\frac{D}{2}\right)} e^{j \phi_{m, n}},
$$

with $K^{\prime}$ the number of real symbol slots per each block. Note that the real symbols are obtained from taking the real and imaginary parts of QAM constellations. Thus, its relation to the symbol slot $K$ introduced in the GFDM system is that $K^{\prime}=2 K$. This also implies that the COQAM and GFDM have a same block length, i.e., $K^{\prime} N_{1}=K M$. The rest of the parameters are in line with those presented in the OQAM scheme. To implement a circular convolution with a prototype filter $g$ of length $L=K M=D+1$, we introduce a pulse shaping filter denoted $\tilde{g}$, obtained by the periodic repetition of duration $K M$ of the prototype filter $g$, i.e., $\tilde{g}[k]=g[\bmod (k, M K)]$.

The COQAM is an orthogonal system and the orthogonality condition for the filter design is analogous to (3), i.e., all the designed prototype filters for OQAM can be reused in COQAM systems. The corresponding baseband COQAM modulation structure is depicted in Figure 5.

\section{Practical implementation schemes}

A direct implementation of (11) cannot be envisioned in practice. In this section two efficient methods are proposed for this implementation. The first one is based on a conventional inverse fast Fourier transform (IFFT), while the second one takes advantage of a pruned IFFT scheme. 


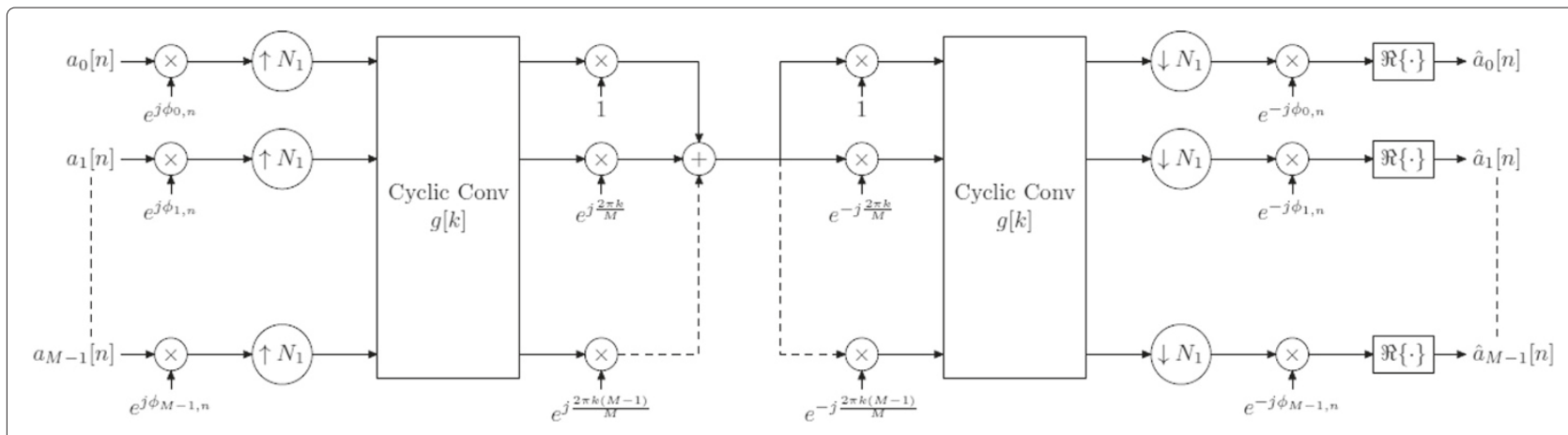

Figure 5 COQAM baseband transmultiplexer.

\subsection{IFFT-based algorithm}

Omitting some mathematical derivations, the baseband COQAM signal, for a block of size $M K$, can be expressed in a matrix form as

$$
\boldsymbol{s}_{M K \times 1}^{\mathrm{COQAM}}=\operatorname{diag}\left\{\boldsymbol{G}_{M K \times K^{\prime}}\left(\boldsymbol{E}_{M K \times M} \boldsymbol{W}_{M \times M} \boldsymbol{A}_{M \times K^{\prime}}\right)^{\mathrm{T}}\right\},
$$

where $(\cdot)^{\mathrm{T}}$ denotes the matrix transpose operation and $\operatorname{diag}\{\cdot\}$ corresponds to the extraction of the diagonal elements of a matrix. $A_{M \times K^{\prime}}$ is the matrix containing the pre-processed data for $m \in[0, M-1]$ and $n \in\left[0, K^{\prime}-1\right]$, i.e.,

$$
\boldsymbol{A}_{M \times K^{\prime}}=\left\{a_{m}[n] e^{j \phi_{m, n}} e^{-\frac{j \pi m D}{M}}\right\}_{M \times K^{\prime}} .
$$

The inverse Fourier transform matrix $\boldsymbol{W}_{M \times M}$, with input indices $(m, k) \in[0, M-1]$, is expressed by $\left\{e^{\frac{j 2 \pi m k}{M}}\right\}_{M \times M}$. The extended matrix $\boldsymbol{E}_{M K \times M}$ corresponds to a $K$ repetition of the identity matrix $\boldsymbol{I}_{M \times M}$ of size $M$ given by

$$
\boldsymbol{E}_{M K \times M}=\left[\begin{array}{lll}
\boldsymbol{I}_{M \times M} & \ldots \boldsymbol{I}_{M \times M}
\end{array}\right]^{\mathrm{T}} .
$$

The polyphase circulant matrix $\boldsymbol{G}_{M K \times K^{\prime}}$ reads as

$$
\boldsymbol{G}_{M K \times K^{\prime}}=\left\{g^{k, n}\right\}_{M K \times K^{\prime}},
$$

with $g^{k, n}=g\left[\bmod \left(k-n N_{1}, M K\right)\right]$, for $k \in[0, M K-1]$ and $n \in\left[0, K^{\prime}-1\right]$.

To get an efficient implementation scheme, the Fourier transform is carried out by a fast IFFT algorithm. Note also that the expansion operation does not require any arithmetic computation. Moreover, as the final operation boils down to the extraction of the diagonal elements, a full computation for matrix multiplications is not necessary. Thus, a large number of computations can be saved. To give a complete picture of our algorithm, we display in Figure 6, the modulation scheme for the processing of

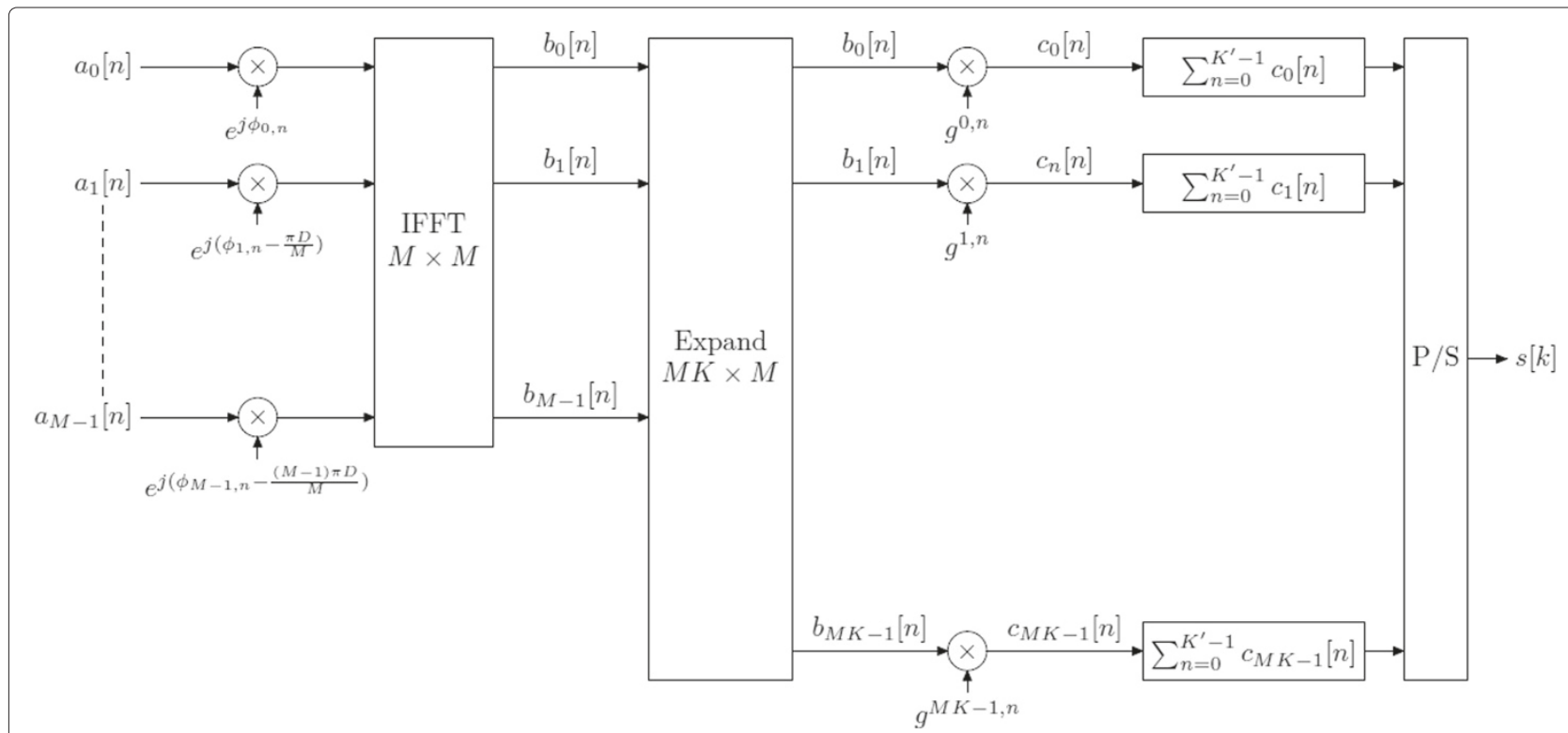

Figure 6 Efficient implementation of the COQAM modulator: IFFT-based algorithm. 
one data block $\boldsymbol{A}_{M \times K^{\prime}}$. It is worth noting that the $g^{k, n}$ coefficients of the prototype filter, shifted for each slot of index $n$, can be deduced from the expression of the prototype filter considering different indexing. Therefore, once $M$ and $K^{\prime}$ are configured, the indexed coefficients can be pre-computed (off-line) and stored in look-up tables for $n=0 \ldots K^{\prime}-1$. Furthermore, the expansion operation in Figure 6, given in (12), is used for our illustration purpose. In practice, there is no need to allocate extra buffers for copying data, one only needs to periodically reuse the same data.

\subsection{Pruned IFFT-based algorithm}

Our second proposal, which further reduces the transmitter complexity, takes advantage of a symmetry property of Fourier Transform. It turns out that only half of the $M$ coefficients at the IFFT output need to be computed. Therefore, a pruned IFFT can be used instead of the conventional $M$-point IFFT, resulting in a reduction of the computational complexity around $50 \%$. A mathematical proof consists in a reformulation of (11) in which we specify the phase term $\phi_{m, n}=\frac{\pi}{2}(m+n)$, i.e.,

$$
s_{\mathrm{COQAM}}[k]=\sum_{n=0}^{K^{\prime}-1} b_{k}^{e}[n] \tilde{g}\left[k-n N_{1}\right], \text { for } k=0 \ldots M K-1,
$$

with

$$
b_{k}^{e}[n]=\underbrace{e^{\frac{j n}{2}} b_{\bmod (k, M)}[n]}_{\text {Permut }+ \text { CYCExt }}, \text { for } k=0 \ldots M K-1,
$$

and

$$
b_{k}[n]=\underbrace{\sum_{m=0}^{M-1} a_{m}[n] e^{-\frac{j \pi m D}{M} e^{\frac{j 2 \pi m(k+M / 4)}{M}}}}_{\text {Pruned IFFT + HSExt }} \text { for } k=0 \ldots M-1 .
$$

The complexity reduction operation involves a pruned IFFT and a Hermitian symmetric extension (HSExt), while a permutation (Permut) and a cyclic extension (CYCExt) are also needed afterwards. Setting $D=L-1=M K-1$, (15) is rewritten as

$$
\begin{aligned}
b_{k}[n] & =\sum_{m=0}^{M-1} a_{m}[n] e^{j \frac{\pi m}{M}} e^{j \frac{2 \pi m(k-M K / 2+M / 4)}{M}}, \\
\text { for } k & =0 \ldots M-1 .
\end{aligned}
$$

Due to the fact that $a_{m}[n]$ are real-valued symbols, the outputs $b_{k}[n]$ do not need to be obtained by a full size IFFT but only a half size, given by

$$
b_{k}[n]=\sum_{m=0}^{M-1} a_{m}[n] e^{j \frac{\pi m}{M}} e^{\frac{j 2 \pi m k}{M}}, \text { for } k=0 \ldots M / 2-1 .
$$

Then the full $M$-point outputs can be completed by the HSExt, i.e.,

$$
b_{M-1-k}[n]=b_{k}^{*}[n], \quad \text { for } k=0 \ldots M / 2-1 .
$$

Next, the Permut renders the right indexing to the outputs, i.e.,

$$
b_{k}[n]=b_{\bmod (k-K M / 2+M / 4, M)}[n], \quad \text { for } k=0 \ldots M-1 .
$$

Last, the CYCExt process completes the necessary $M K$ point data for prototype filtering, for $k=0 \ldots M K-1$, given by

$$
b_{k}^{e}[n]= \begin{cases}b_{\bmod (k, M)}[n], & n=2 q \text { with } q \text { even } \\ -b_{\bmod (k, M}[n], & n=2 q \text { with } q \text { odd } \\ j b_{\bmod (k, M)}[n], & n=2 q+1 \text { with } q \text { even } \\ -j b_{\bmod (k, M)}[n], & n=2 q+1 \text { with } q \text { odd }\end{cases}
$$

Assuming $M$ is a power-of-2 integer, the traditional $M$-point IFFT, using the radix-2 Cooley-Tukey algorithm [35], requires $(M / 2) \log _{2} M$ complex multiplications. Then, a pruned IFFT transform with $M$ inputs and $N_{1}$ outputs $\left(N_{1}=M / 2\right)$ yields a complexity reduction of at least $50 \%$ [36], i.e., $(M / 4) \log _{2} M$. Although it is not detailed in this paper, in practice, one could further imagine to design a filter with odd-value filter length, e.g., SRRC. With this change, it can be readily derived that the pre-processing in Figure 7 is completely avoided, leading the arithmetic complexity, for generating one COQAM block of size $M K$, to

$$
\text { \# mult. }=\underbrace{K(M / 2) \log _{2} M}_{\text {OFDM }}+M K^{2} .
$$

In (16), the former part reflects the number of multiplications needed for OFDM modulation, processing the same amount of the data symbols. Thus, $M K^{2}$ represents the additional arithmetic complexity. In fact, the exact complexity calculation depends on the implementation algorithm. For example, the algorithm presented in this section is one of the possible ways to implement COQAM system. One may also consider using a frequency domain implementation concept which is introduced for GFDM and CB-FMT. Thus, the resulting complexity differs as a function of implementation algorithms. Moreover, the algorithm preference must be analyzed in the context of concrete system parameter setting, e.g., frame structure and concrete $M$ and $K$ values.

\section{COQAM transceiver design in a radio system}

In order to maintain a perfect orthogonality after a transmission through a multi-path channel, we introduce a CP to cancel the inter-block interference. Moreover, as we want to prevent an alteration of the PSD resulting from a spectral leakage due to the block processing, a windowing is applied after $\mathrm{CP}$ insertion. The transmission system resulting from these two operations is named windowed 


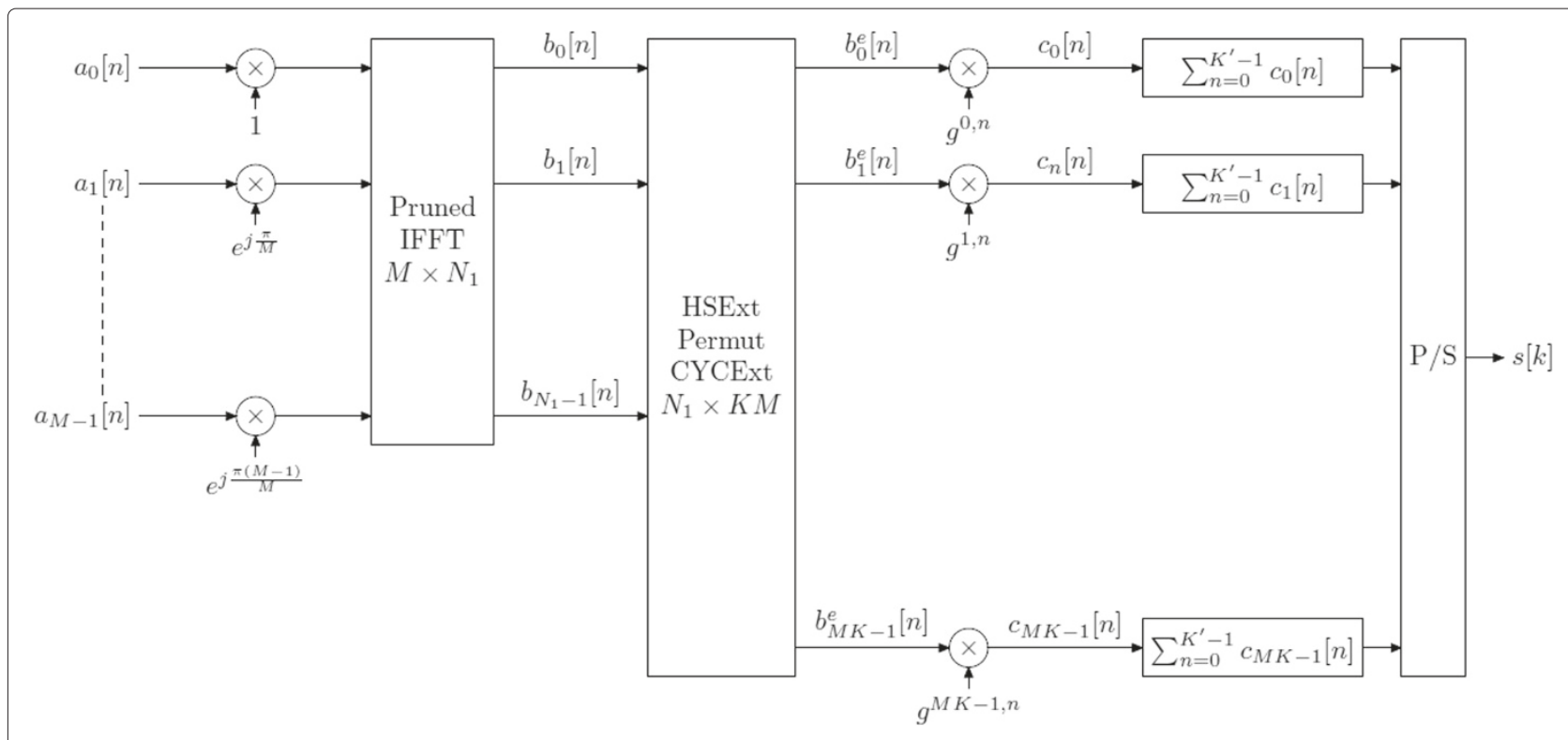

Figure 7 Efficient implementation scheme of the COQAM modulator: pruned IFFT-based algorithm.

CP-COQAM (WCP-COQAM). Denoting the CP length by $L_{\mathrm{CP}}$, we get

$$
L_{\mathrm{CP}}=L_{\mathrm{GI}}+L_{\mathrm{RI}} \text {, }
$$

where $L_{\mathrm{GI}}$ is the CP part used to fight against the multipath channel interference and $L_{\mathrm{RI}}$ is the portion devoted to windowing.

The $l$ th block of the WCP-COQAM signal $s_{\text {WCP-COQAM }}[k]$, for $k=0 \ldots M K+L_{\mathrm{CP}}-1$, can be obtained from the $l$ th block of the COQAM signal by

$$
\begin{aligned}
s_{\text {WCP-COQAM }}[k]= & \sum_{r=l-1}^{l+1} s_{\text {COQAM }}\left[\bmod \left(k-L_{\mathrm{CP}}, M K\right)\right] \\
& \times w[k-r Q],
\end{aligned}
$$

where $Q=M K+L_{\mathrm{GI}}$ and $w[k]$, defined in the $k=$ $0, \ldots, M K+L_{\mathrm{CP}}-1$ interval, is the window function computed as follows

$$
w[k]= \begin{cases}\text { window coeffs. } & k \in\left[0, L_{\mathrm{RI}}-1\right] \\ 1 & k \in\left[L_{\mathrm{RI}}, M K+L_{\mathrm{GI}}-1\right] \\ w\left[M K+L_{\mathrm{CP}}-1-k\right] & \text { otherwise. }\end{cases}
$$

There are extensive works on the window design [31]. In this paper, we simply use the triangle (Fejer, Bartlet) window for this windowing process. Particular investigation on the window design in the context of COQAM will be envisaged in a future step. Furthermore, it is worth noting that the additional part $L_{\mathrm{RI}}$ does not reduce the spectral efficiency since consecutive blocks are overlapped with the RI part, which means that the true redundancy for the transmission is the GI part. Moreover, it will be illustrated later that on the receiver side, for each block, only the first
GI samples are removed. The WCP-COQAM transmitter structure is depicted in Figure 8, where $s_{1}[k]$ denotes $s_{\text {COQAM }}[k]$, which is obtained from (11) and $s_{2}[k]$ stands for $s_{\text {WCP-COQAM }}[k]$.

Regarding the receiver structure, there are several distinct algorithms for the WCP-COQAM demodulation. In this paper, we present one algorithm that is similar to the one presented for GFDM [17] and CB-FMT [24]. The structure is depicted in Figure 9 and a detailed illustration is given in the following. Assuming a perfect synchronization, the received WCP-COQAM signal is passed through a pre-processing phase, which takes away the $\mathrm{CP}$ and the windowing effect. This process consists in two steps. The received discrete signal is stored in a buffer of size $L+L_{\mathrm{GI}}$ samples $(L=M K)$. Then, the first $L_{\mathrm{GI}}$ samples are omitted and followed by a cyclic shift operation, which can be written as

$$
s[k]=s\left[\bmod \left(k+L_{\mathrm{GI}}-1, M K\right)\right], \text { for } k=0 \ldots M K-1 .
$$

Next, an $L$-point FFT operation is performed so that a frequency domain equalization (FDE) can be straightforwardly envisaged. Indeed, different equalization criteria can be chosen, e.g., minimum mean square error (MMSE) [24]. Here we stick to a simple one-tap ZF equalization. After, the $L$ outputs are multiplied by a shifted frequency domain prototype filter coefficient $G^{q, m}$, whose coefficients are computed with an $L$-point Fourier transform of the prototype filter $g[k]$ and shifted with regard to the subcarrier index $m$, i.e., for $q=0 \ldots M K-1$ and $m=$ $0 \ldots M-1$, we have

$$
G^{q, m}=G\left[\bmod \left(q+n K^{\prime}, M K\right)\right],
$$




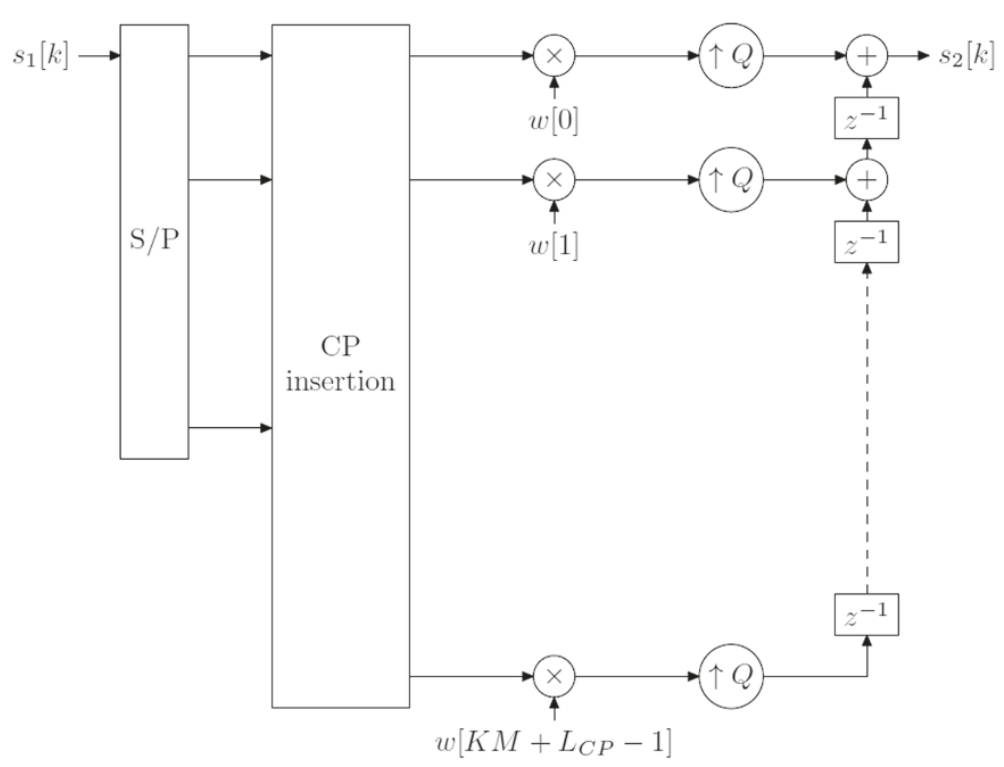

Figure 8 Transmitter of the WCP-COQAM system.

with

$$
G[q]=\sum_{k=0}^{M K-1} g[k] e^{-j \frac{2 \pi k q}{M K}} .
$$

Afterwards, a folder operation is repeatedly performed for each of the $M$ subcarriers which indeed realizes a subblock summation, i.e.,

$$
\boldsymbol{E}_{M K \times K^{\prime}}^{\mathrm{T}}=\left[\boldsymbol{I}_{K^{\prime} \times K^{\prime}} \ldots \boldsymbol{I}_{K^{\prime} \times K^{\prime}}\right]^{\mathrm{T}} .
$$

Note that a lot of computation savings can be envisioned here, because a large number of the coefficients of $G[q]$ are trivial values. Finally, a $K^{\prime}$-point IFFT is performed for each subcarrier and the post-phase processing is given by

$$
\psi_{m, n}=-\left(\frac{\pi}{2}(m+n)-\frac{\pi m}{M}\left(D-N_{1}+2\right)\right) .
$$

The detailed processing diagrams for the WCP-COQAM receiver structure are illustrated in Figure 9. The complexity analysis of the WCP-COQAM system will be discussed later in this paper.

\section{Performance evaluation}

Our research objective is to find an enhanced MCM scheme to maximally address the considered four aspects.

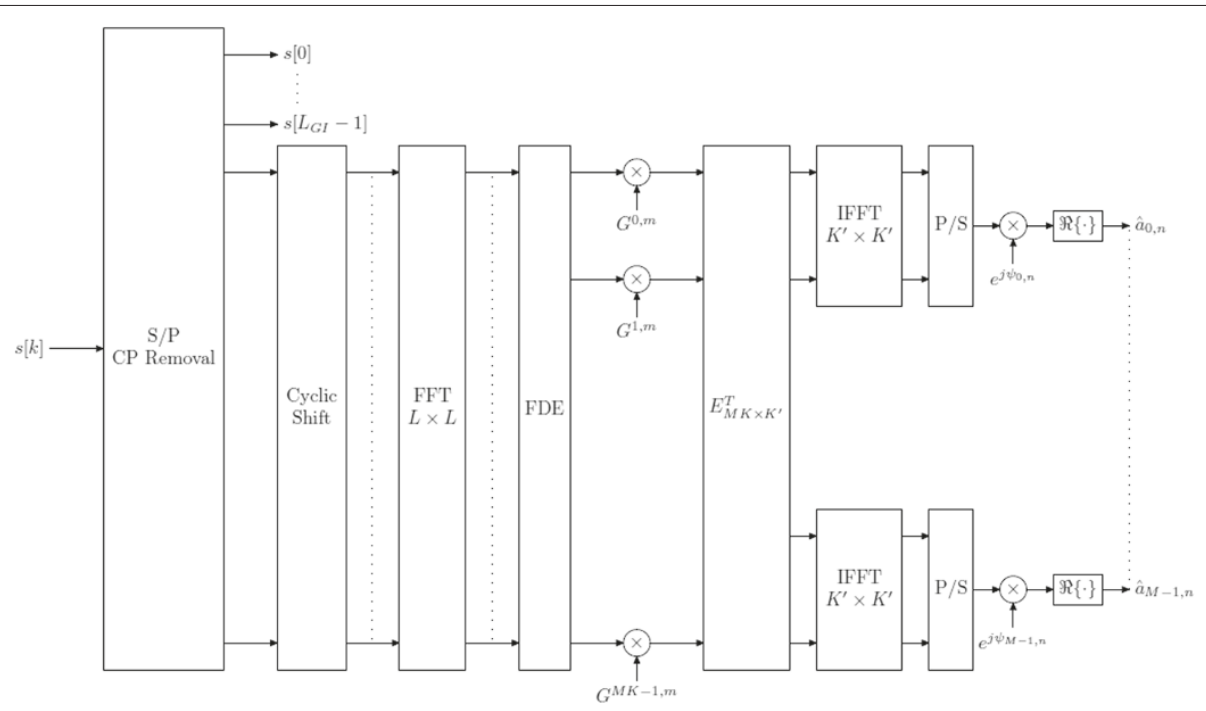

Figure 9 WCP-COQAM receiver structure. 
Therefore, we repeat the same analysis that has been reported in Section 2.2. This time, we take the WCPCOQAM into the comparison. In addition, the Bit Error Rate (BER) vs. Signal-to-Noise Ratio (SNR) comparison is also given for further illustrating the efficiency of the proposal in the presence of the background noise.

\subsection{Spectral efficiency evaluation}

First off, we analyze the SE of WCP-COQAM. As illustrated in Section 5, for each WCP-COQAM block of size $M K$ samples, a $C P$ is inserted in the front of each block and is composed of two parts, i.e., $L_{\mathrm{GI}}$ and $L_{\mathrm{RI}}$. However, the latter part does not reduce the SE. Therefore, assuming that $L_{\mathrm{GI}}$ has the same quantity as that used for the GFDM, the spectral efficiency indicator of the WCP-COQAM is same as that of the GFDM systems, i.e.,

$$
\mathrm{SEI}_{\mathrm{WCP}-\mathrm{COQAM}}=\frac{1}{\left(T_{0}+T_{\mathrm{GI}} / K\right) \cdot F_{0}}=\frac{T_{0}}{T_{0}+\frac{T_{\mathrm{GI}}}{K}}<1 .
$$

\subsection{Power spectral density evaluation}

In the respect of PSD evaluation, the SRRC filter is also used for the WCP-COQAM scheme. The better frequency-localized feature of SRRC pulse naturally makes the WCP-COQAM outperform the CP-OFDM, while the potential spectral leakage due to its block transform processing may augment the out-of-band radiation. This problem can be overcome with our proposed windowing process, and the estimated PSD in Figure 10 confirms this statement. Thus, the WCP-COQAM can maintain an excellent PSD shape. In addition, to effectively enable future DSA solutions, the signal PSD in fragmented

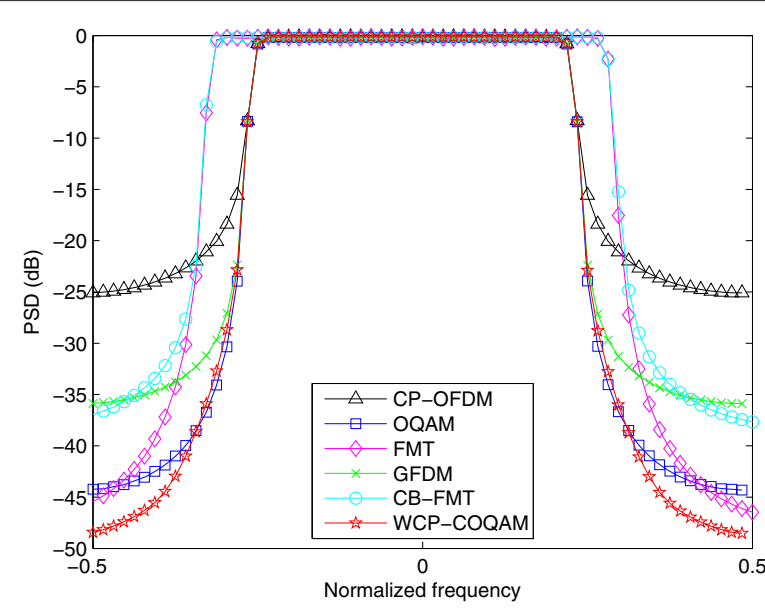

Figure 10 PSD evaluation of our proposed windowing process. $M=64$ and $M / 2$ active carriers; CP length $M / 4$ for CP-OFDM, GFDM and CB-FMT; SRRC filter of roll-off factor 0.2 with overlapping factor 32 for OQAM, FMT, GFDM, CB-FMT, and WCP-COQAM; $N_{2}=(5 / 4) M$ for FMT and CB-FMT; $K=32$ for GFDM and CB-FMT; $L_{G I}=M / 4$ and $L_{R I}=6$ for WCP-COQAM bands should also be evaluated because in future systems, non-contiguous frequency band allocation will be an inevitable choice to drive the DSA to its ultimate limit. For this reason, Figure 11 shows the PSD comparison in the fragmented band case, where we switch off the same number of subcarriers located in the middle band for all these schemes. The result shows that the WCP-COQAM can still keep a satisfactorily low radiation level in the notch band.

\subsection{Why is circular convolution necessary?}

WCP-COQAM transforms the continuous-processing to the block-processing such that with the aid of $\mathrm{CP}$, the onetap frequency domain equalization can become effective. Nevertheless, one may wonder if the circular filtering is really necessary. For the classical OQAM scheme, it can also introduce a CP then applies the frequency domain equalization at the receiver. Such scheme is named CPOQAM and it was proposed in [37]. In this section, we explain the necessity of using circular filtering instead of linear filtering and the difference between WCP-COQAM and CP-OQAM.

From the robustness against multi-path interference point of view, both schemes introduce $\mathrm{CP}$ and can ensure an interference-free transmission. Therefore, at this point, WCP-COQAM and CP-OQAM are quite equivalent. Nevertheless, for WCP-COQAM, there does not exist any waveform discontinuity within one block even when the $\mathrm{CP}$ is inserted. The only discontinuity appears between two consecutive blocks, which can be perfectly removed by the presented windowing process. On the other hand, for CP-OQAM, if a simple cutting is used to define blocks [37], the waveform is cut into two or several blocks depending on the prototype filter length and block length. In this case, the waveform discontinuity occurs within

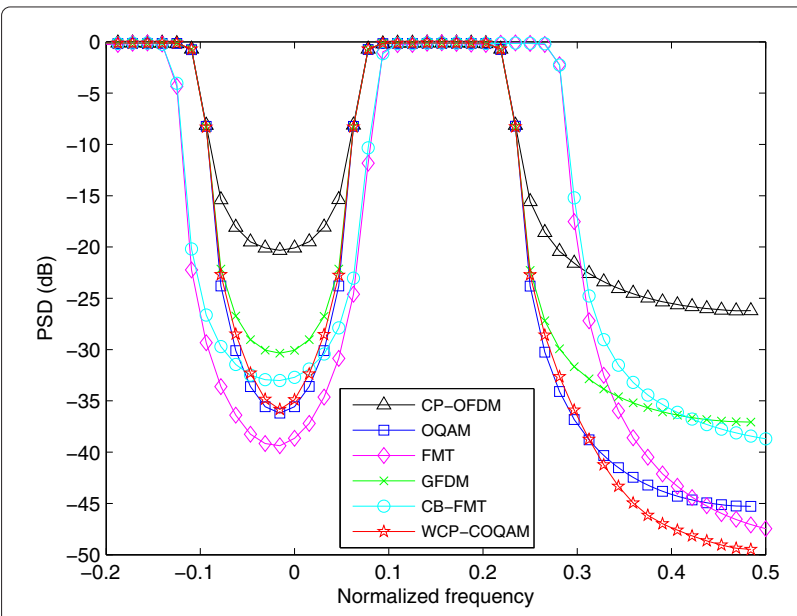

Figure 11 PSD evaluation in fragmented band case: same parameter setting as Figure 10; 10 subcarriers are switched off. 
the block especially when $\mathrm{CP}$ is appended in front of each block. Moreover, such discontinuity cannot be alleviated by the windowing process, as they do not appear on the block edges. In Figure 12, we show the PSD comparison between WCP-COQAM and CP-OQAM (w./wo. windowing) to prove the above statement. It is clearly shown that for CP-OQAM the discontinuity issue cannot be solved by the windowing process.

\subsection{Orthogonality evaluation}

The orthogonality evaluation for the WCP-COQAM is also done for E2E and MP cases. Same parameter setting as in Section 2.2.3 is used here. The EVM comparison in the E2E case is reported in Figure 13, where the WCPCOQAM curve is quite similar to the OQAM curve. This proves that the WCP-COQAM with SRRC filter can form a nearly perfect reconstruction system. Unlike OQAM, when we now move to the MP case, shown in Figure 14, the WCP-COQAM can still maintain the best orthogonality, without being impaired by the time-frequency localization property of the prototype filter. This also indicates that the WCP-COQAM can indeed employ flexible waveforms without sacrificing this orthogonality feature.

To provide a full picture of the orthogonality analysis, we further show the BER vs. SNR comparisons for all these MCM schemes. The constellation order is set to 16QAM; we continue to use the simple two-tap channel as in Section 2.2.3. Figures 15,16,17 give the BER comparison results for different MCM schemes in three cases of SRRC filter with RO factor being $0.2,0.5$, and 0.8 , respectively. The CP-OFDM performance is also included this time and it can be referred as a baseline in our comparison. Note that the SNR is considered by the total power spent on the transmitted symbols without taking into account the SE. The reason for this is to make the CP-OFDM an

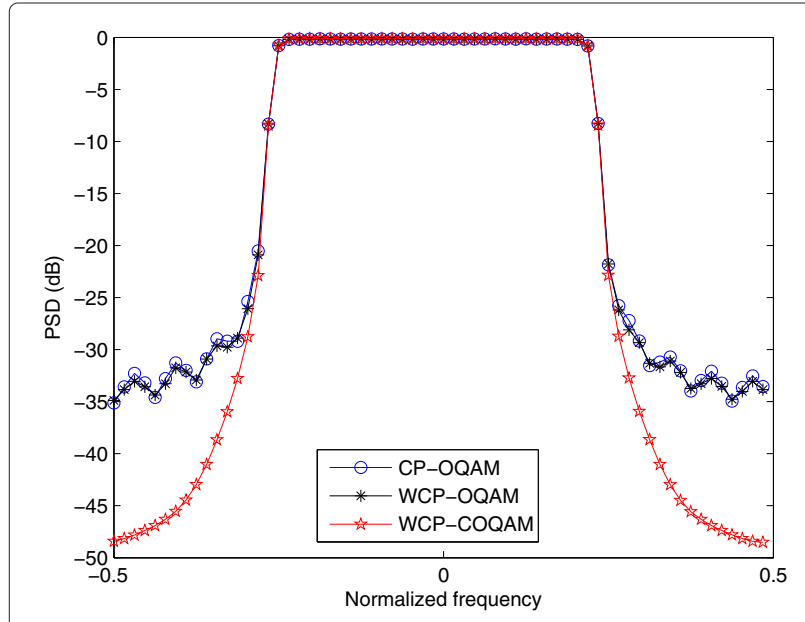

Figure 12 PSD comparison WCP-COQAM vs. CP-OQAM vs. WCP-OQAM: same parameter setting as Figure 10.

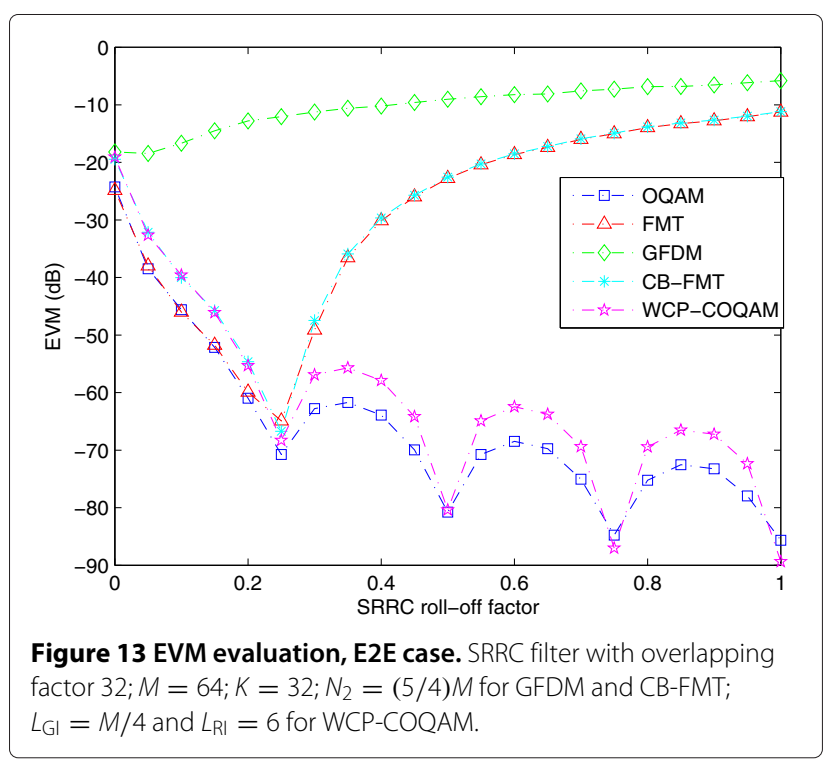

accurate reference reflecting the interference-free performance. Nevertheless, in Table 1, the power loss due to $\mathrm{CP}$ insertion and subcarrier spacing increase (i.e., oversampling) is given for each MCM scheme. The results show a good match between WCP-COQAM and CPOFDM. The performance improvement, compared with OQAM, is clear and will be even more significant when the constellation order continues to increase. This proves that the drawback of OQAM is overcome by the WCPCOQAM scheme. Moreover, as the RO factor varies from 0.2 to 0.8 , the BER curves of WCP-COQAM always matches with those of CP-OFDM. This further confirms

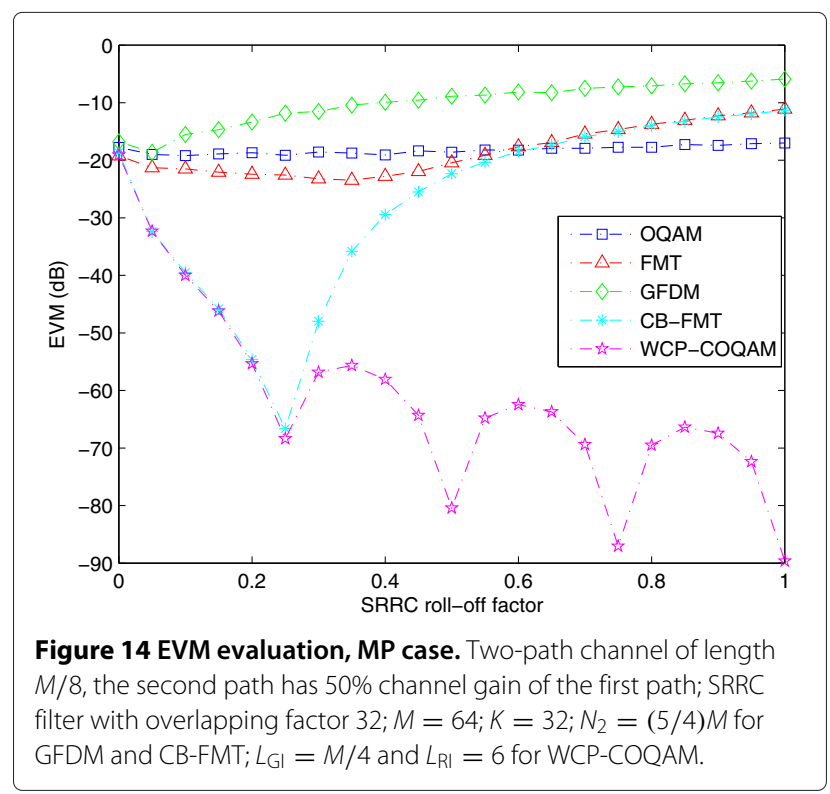




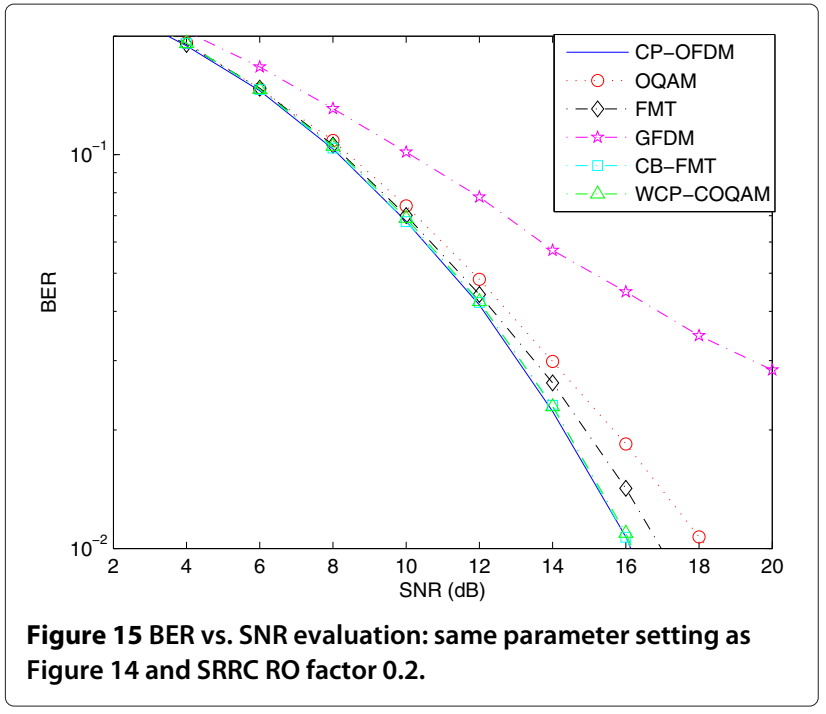

that the orthogonality feature of the WCP-COQAM system is not impacted by the waveform flexibility, which is the crucial factor for future mobile radio systems. On the other hand, when RO factor tends to a large value, in the BER performances of FMT and CB-FMT a severe degradation appears, which is in line with the EVM analysis. Finally, the GFDM results in the worse BER performance due to its non-orthogonality. Therefore, we can deduce that it probably is impossible to function a GFDM-based system with a one-tap equalization.

To give bird's eye view on all of these MCM schemes evaluation, in Figure 18, we introduce a pentagon-shaped metric, whose five corners represent five evaluation criteria, i.e., SE, PSD, waveform flexibility (WF), orthogonality in E2E (OrE2E), and MP (OrMP) cases. Moreover, we use the inner/middle/outer layers to indicate three robustness

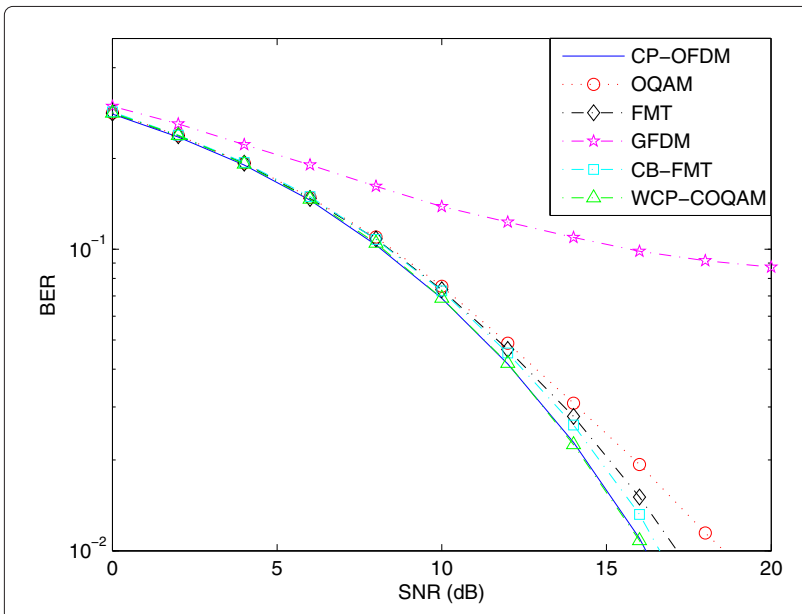

Figure 16 BER vs. SNR evaluation: same parameter setting as Figure 14 and SRRC RO factor 0.5 .

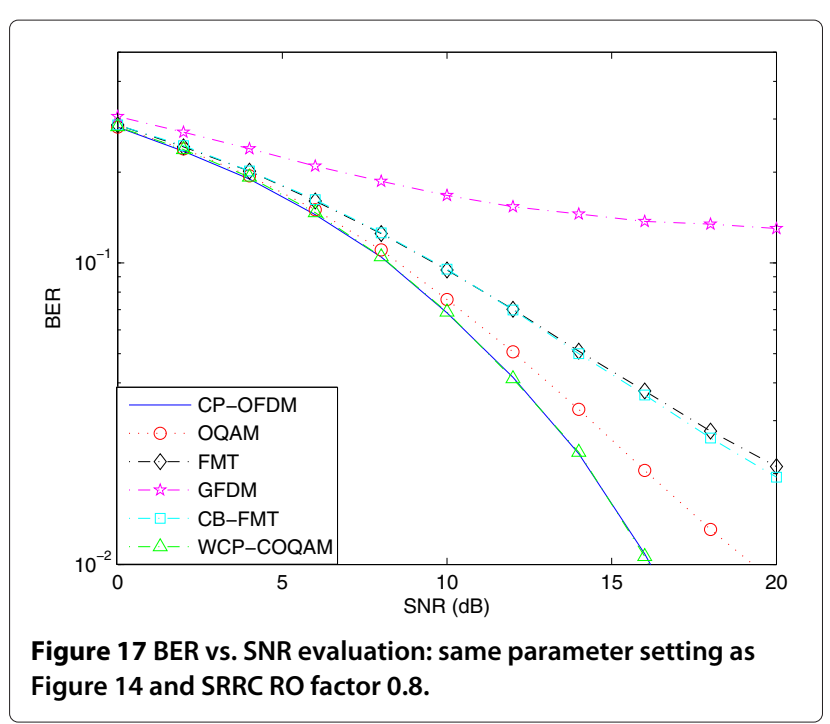

levels, i.e., poor/average/good. The corresponding curve of each MCM scheme reflects the final evaluation score, which gives the preference of the suitable scheme for future radio systems. Clearly, the WCP-COQAM minimizes the predecessors' drawbacks while still keeping the maximum number of the advantages.

\section{Discussions}

\subsection{WCP-COQAM receiver complexity}

Similar to the transmitter side, the receiver complexity is also of paramount importance to be evaluated. Here, we provide a simple complexity analysis in terms of the number of arithmetical computations, i.e., complex multiplications $(\mathrm{CM})$. Then we provide a complexity and performance comparison of WCP-COQAM vs. the classical OQAM receiver employing non-trivial equalizers. This comparison reveals the interest of using WCP-COQAM scheme.

For WCP-COQAM receiver as shown in Figure 9, the arithmetical computation for processing one block of the received data ( $L=M K$ samples) can be divided into the computation of one FFT of size $L$, FDE for one block, polyphase filtering (product of FDE outputs and $G^{q, m}$ ), and IFFTs of size $K^{\prime}$ for $M$ subcarriers, noting that the

Table 1 Power loss due to CP and over-sampling

\begin{tabular}{ll}
\hline & Power loss $(\mathbf{d B})$ \\
\hline CP-OFDM & 0.97 \\
OQAM & 0 \\
FMT & 0.97 \\
GFDM & 0.03 \\
CB-FMT & 1 \\
WCP-COQAM & 0.03 \\
\hline
\end{tabular}




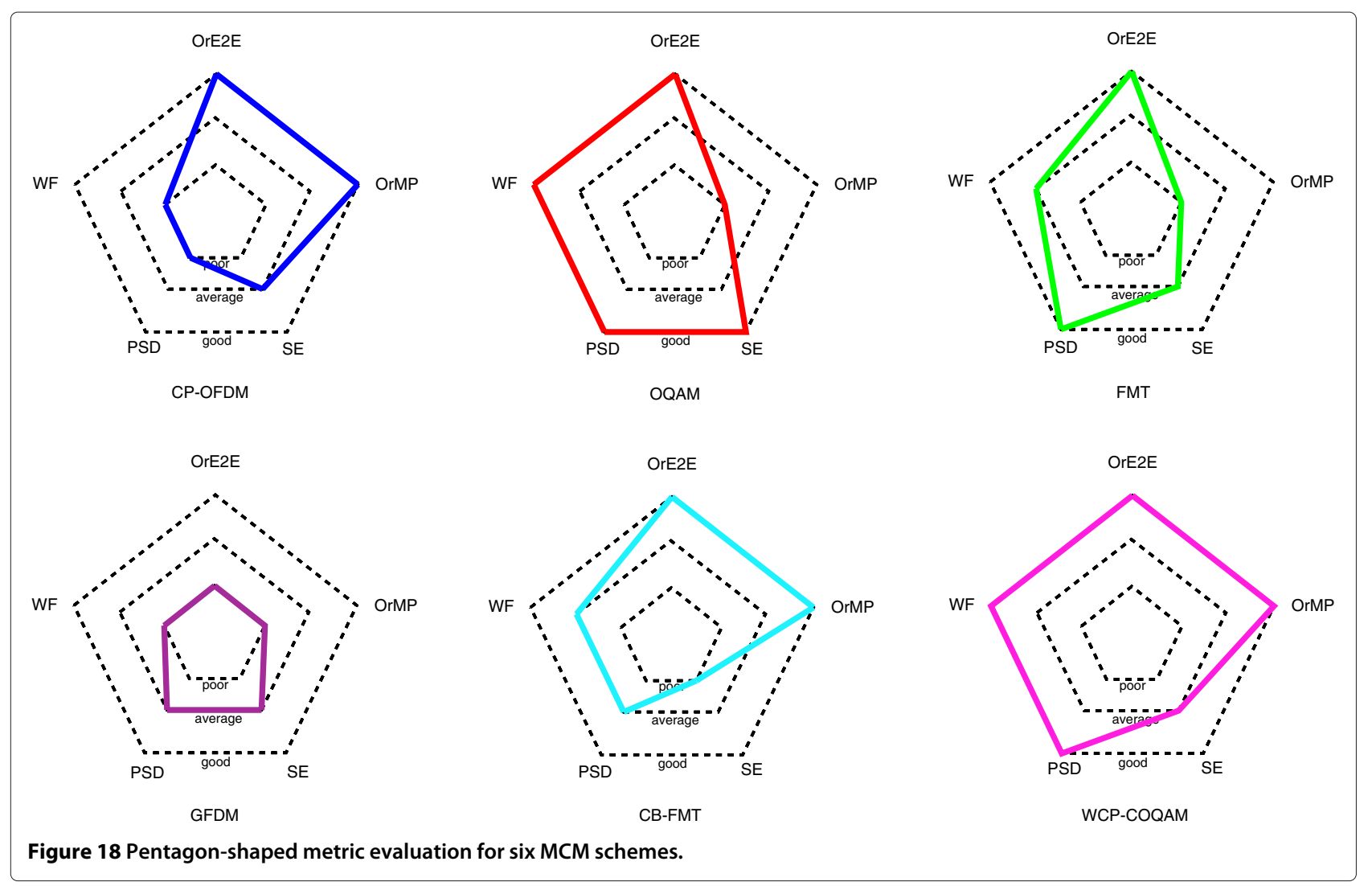

cyclic shift operation does not consume any arithmetical computations. Here we do not count the post-phase processing as it does not represent additional complexity with regard to the classical OQAM receiver. In more detail, the $L$-point FFT needs $(L / 2) \log _{2} L$ CMs. The FDE, which represents one-tap equalizer, yields $L$ CMs. The product between FDE outputs and $G^{q, m}$ is a special case. As $G^{q, m}$ represents the shifted version of the frequency coefficients of the prototype filter, it turns out that only a small portion of the frequency coefficients are non-zero-valued. If one uses the SRRC filter, the number of the non-zero coefficients can be analytically calculated. Assuming a SRRC prototype filter with RO factor $r$, its bandwidth thus yields $\frac{1+r}{M T_{\mathrm{s}}}(\mathrm{Hz})$, where $T_{\mathrm{s}}$ stands for the sample duration. From (19) it shows that frequency resolution for calculating the frequency coefficients is $1 / M K T_{\mathrm{s}}(\mathrm{Hz})$. Therefore, the number of non-zero coefficients can be obtained with the division between the bandwidth and the frequency resolution, i.e., $\lceil(1+r) K\rceil$ with $\lceil\cdot\rceil$ being the ceiling operation. Thus, the polyphase filtering consumes $M\lceil(1+$ $r) K\rceil \mathrm{CMs}$. At last, each of the $K^{\prime}$-point IFFT results in $\left(K^{\prime} / 2\right) \log _{2} K^{\prime}=K \log _{2} 2 K$ CMs which lead to a total of $M K \log _{2} 2 K$ CMs.

Regarding the classical OQAM receiver for processing the same amount of data, we look at the two cases. The first uses a two-step equalizer named equalization with interference cancelation (EIC) [13], and the second one applies a multi-tap equalizer (MTE) [32]. Assuming that the prototype filter is of length $M K$, the arithmetical computation needed for classical OQAM demodulation yields $K^{\prime}$ times $M$-point FFT ( $M K \log _{2} M \mathrm{CMs}$ ), and the polyphase network (PPN) consumes $M K^{2}$ CMs. In the case of EIC, the on-line arithmetical computation ${ }^{\mathrm{e}}$ [13] yields $\left(8 \kappa^{2}+8 \kappa+2\right) M K$ CMs where $\kappa$ stands for the neighborhood zone within which the interference is considered for the interference canceler. In the case of multi-tap equalizer, the equalization yields $2 \eta M K$ CMs, where $\eta$ represents the number of the taps. Table 2 summarizes the analyzed complexity for further comparison.

As explained in Section 2.2.2, the SRRC filter was chosen in our previous performance evaluation to ensure a fair comparison among different MCM schemes. However, the drawback of SRRC filter comes from the necessity of using a long filter length which results in

Table 2 Receiver complexity analysis

\begin{tabular}{ll}
\hline Schemes & Number of complex multiplications \\
\hline WCP-COQAM & $\left.M K\left(\frac{\log _{2} M K}{2}+\log _{2} 2 K+1\right)+M \Gamma(1+r) K\right\rceil$ \\
OQAM-EIC & $M K \log _{2} M+M K^{2}+\left(8 \kappa^{2}+8 \kappa+2\right) M K$ \\
OQAM-MTE & $M K \log _{2} M+M K^{2}+2 \eta M K$ \\
\hline
\end{tabular}


a high receiver complexity. In practice, for the WCPCOQAM and classical OQAM schemes, the orthogonality condition (3) can be perfectly held with a moderate filter length, e.g., overlapping factor $\leq 4$. In what follows, we show the performance comparison among WCP-COQAM, OQAM-EIC, and OQAM-MTE.

In this simulation, the prototype filter is now changed to a OQAM-dedicated filter named frequency selective (FS) [27] with overlapping factor of 4. This corresponds for WCP-COQAM to $K=4$. For OQAM-EIC, the neighborhood zone is set to 1 , indicating that only the interference coming from the closest neighbors is canceled. For OQAM-MTE, a three-tap equalizer is considered. Moreover, we increase the channel delay spread up to $M / 6$ in order to clearly show the performance degradation due to the multi-path interference. The rest of the parameters are in line with those reported in Section 6. The performance is reported in Figure 19 and the corresponding power loss and the complexity are given in Tables 3 and 4, respectively. Note that our FS filter is designed to have similar bandwidth to SRRC with RO factor 1 . The results confirm that the WCP-COQAM can address a better performance with less complexity.

\subsection{How can WCP-COQAM combine with Alamouti?}

As discussed in the previous section, the WCP-COQAM has similar robustness of the multi-path interference immunity as for the CP-OQAM scheme. Therefore, the solutions proposed for CP-OQAM scheme can be easily tailored to WCP-COQAM system. Among them, one solution called pseudo-Alamouti encoding was proposed to CP-OQAM [38] which allows for a simple low-complexity decoding algorithm on the receiver side. To extend this scheme for WCP-COQAM, the encoding process can remain the same as [38], while the receiver

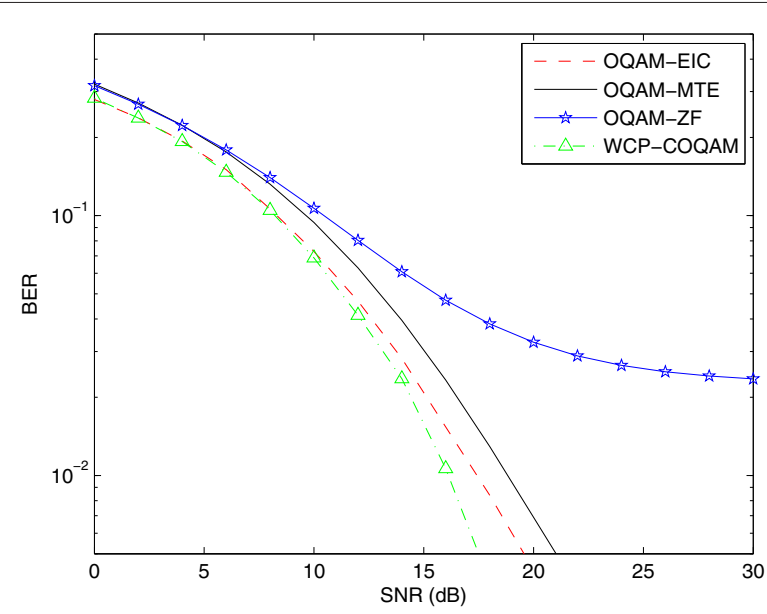

Figure 19 BER vs. SNR evaluation: WCP-COQAM vs. OQAM-EIC vs. OQAM-MTE.
Table 3 Power loss due to CP

\begin{tabular}{ccccc}
\hline & WCP-COQAM & OQAM-ZF & OQAM-EIC & OQAM-MTE \\
\hline Power loss & $0.26 \mathrm{~dB}$ & $0 \mathrm{~dB}$ & $0 \mathrm{~dB}$ & $0 \mathrm{~dB}$ \\
\hline
\end{tabular}

structure should be modified according to Figure 9. The details of the WCP-COQAM-based Alamouti transmission will be given in our future publication. Moreover, in the literature, there are proposals on the AlamoutiFBMC/OQAM, e.g., [39,40]. In [39], a block-wise Alamouti encoding is proposed, where the notion of block is defined by inserting zero-symbols, leading to a compromise between the spectral efficiency and receiver memory buffer. In [40], an FFT-FBMC scheme, together with a special data transmission strategy has been proposed. This scheme can be seen as a subcarrier-wise precoded FBMC system. The ISI at each subcarrier can be easily removed by a simple equalization, thanks to the subcarrier-wise IFFT precoding and CP insertion, while the ICI level can be controlled by a special data transmission strategy and good frequency-localized prototype filter. It is claimed that this scheme can well enable the Alamouti transmission with a reasonable added complexity. The performance evaluation as well as the complexity comparison among these schemes for Alamouti transmission will be envisioned in our future work.

\subsection{WCP-COQAM receiver flexibility}

It is worth pointing out that there are potential degrees of flexibility that can be explored on the WCP-COQAM receiver side. In fact, as stated in Section 5, the presented receiver structure in Figure 9 illustrates one possible way of receiver processing. Otherwise said, it can be considered as one receiver mode. Alternative receiver modes can be envisioned, for instance, we can imagine to establish a receiver structure which realizes a dual process to Figure 6, as shown in Figure 20, where this mode performs the equalization after the filter bank. Moreover, the SoTA equalization methods for classical OQAM can be readily adapted to this mode which could be more beneficial in some particular transmission contexts, e.g., high Doppler spread. The most interesting feature is that this receiver flexibility leaves the receiver to decide which mode to be activated without any transmitted signal modifications. It indeed inspires future research investigations on how to efficiently take advantage of this flexibility.

Table 4 Complexity comparison in a specific context

\begin{tabular}{lc}
\hline Schemes & Number of complex multiplications \\
\hline WCP-COQAM & 2,560 \\
OQAM-EIC & 7,168 \\
OQAM-MTE & 4,096 \\
\hline
\end{tabular}




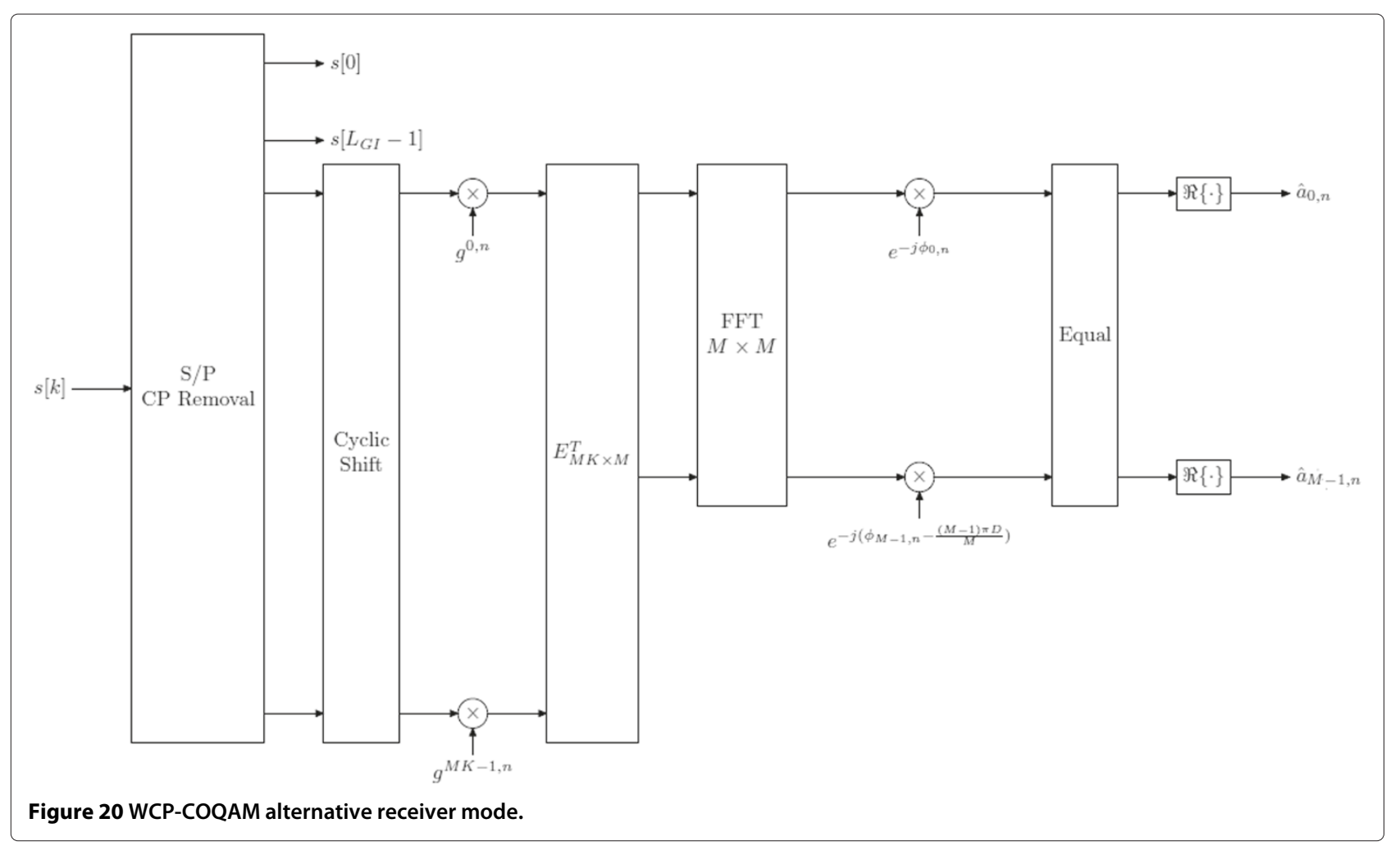

\subsection{Differentiation of WCP-COQAM and Abdoli et al.}

In the end, we would like to spend some space to discuss the commonality and the difference between our contributions and that of in [41]. First off, [41] has a different motivation that aims at the reduction of the overhead of the OQAM system in a burst transmission, which ends up with a similar concept that uses a circular convolution filtering instead of the linear convolution, and a time-windowing is also proposed to improve the PSD. Nevertheless, the differences between the proposal of [41] and ours are that our proposal is based on a regular circular convolution, while in [41] it is claimed that the regular circular convolution is avoided and instead, a weighted circular convolution version is used. Second, the system realization is totally different and the windowing process is also different. Moreover, the orthogonality enhancement in a multi-path channel context is not in the investigation scope of [41]. Finally, comparing the classical OQAM scheme, with the same SRRC prototype filter the proposal in ([41], Tab. I), leads to an orthogonality loss, while this is not the case in our proposal.

\section{Conclusions}

In this paper, we analyzed five SoTA MCM schemes from different aspects which are deemed as the important factors for future radio systems. The analyzed results showed that none of the SoTA schemes can simultaneously address these aspects. Then, we proposed a novel MCM scheme, which is a combination version of the classical OQAM with the circular convolution concept. Its concept and efficient implementation as well as its transceiver algorithm were reported. Finally, the efficiency of the proposed scheme was analyzed in terms of the evaluation criteria, and it turned out that our proposed scheme can indeed address these aspects to a maximum degree. Nevertheless, the selected evaluative criteria do not cover all of the research aspects that naturally triggers further investigations. To continue the work, our future research will cover the performance and complexity analysis for Alamouti-encoded transmission, in which different proposals in the literature will be compared together. Moreover, the sensitivity of WCP-COQAM to the synchronization error will also be addressed. Furthermore, effort will also be made to address the remaining issues such as how to adapt the pilot structure to the WCP-COQAM system and how to take advantage of the WCP-COQAM receiver flexibility.

\section{Endnotes}

${ }^{a}$ During the paper preparation, we spotted that a similar idea was independently investigated in [41] A discussion will be given at the end of this paper to differentiate the different contributions between our proposal and [41]. 


\author{
${ }^{\mathrm{b}}$ In this paper, the PSD is simulated with Welch's \\ method, where the segment length is set to $M$ for \\ CP-OFDM, OQAM, and GFDM and $N_{2}$ for FMT and \\ CB-FMT. The initial shift between segments is taken \\ equal to half of the segment length.
}

${ }^{\mathrm{C}}$ In the literature, the only reported filter for the GFDM and CB-FMT systems is the SRRC. Therefore, in this paper, we stick to the SRRC to ensure the filter alignment, even though it is not the most favorable choice for the OQAM and FMT schemes as it cannot exactly hold an orthogonal condition with a finite length.

${ }^{\mathrm{d}} \mathrm{A}$ condition for this statement is that the CP-OFDM symbol duration is smaller than the channel coherence time.

${ }^{\mathrm{e}}$ On-line arithmetical computation means the calculation needed for each received sample. Its counterpart is the periodic mathematical computation, meaning that the calculation is only needed punctually.

\section{Competing interests}

The authors declare that they have no competing interests.

\section{Acknowledgements}

This work has been performed in the framework of the FP7 project ICT-317669 METIS. The authors would like to acknowledge the contributions of their colleagues.

\section{Received: 22 November 2013 Accepted: 6 May 2014}

Published: 30 May 2014

\section{References}

1. METIS deliverable D6.2, Initial report on horizontal topics, results and system concept. Technical Report ICT-317669 (March 2014)

2. METIS deliverable D1.1, Scenarios, requirements and KPIs for $5 \mathrm{G}$ mobile and wireless system. Technical Report ICT-317669 (May 2013)

3. G Matz, H Bölcskei, F Hlawatsch, Time-frequency foundations of communications. IEEE Signal Process. Mag. 30(6), 87-969 (2013)

4. H Saeedi-Sourck, Y Wu, JWM Bergmans, S Sadri, B Farhang-Boroujeny, Sensitivity analysis of offset QAM multicarrier systems to residual carrier frequency and timing offsets. Signal Process. 91(7), 1604-1612 (2011)

5. H Lin, M Gharba, P Siohan, Impact of time and carrier frequency offsets on the FBMC/OQAM modulation scheme. Signal Process. 102, 151-162 (2014)

6. JJ Benedetto, C Heil, DF Walnut, Differentiation and the Balian-Low theorem. J. Fourier Anal. Appl. 1(4), 355-402 (1995)

7. BR Saltzberg, Performance of an efficient parallel data transmission system. IEEE Trans. Commun. Technol. 15(6), 805-811 (1967)

8. SB Weinstein, PM Ebert, Data transmission by frequency-division multiplexing using the discrete Fourier transform. IEEE Trans. Commun. 19, 628-634 (1971)

9. R Vallet, K Haj Taieb, Fraction spaced multi-carrier modulation transmission. Wireless Pers. Commun. 2, 97-103 (1995)

10. R Hleiss, P Duhamel, M Charbit, Oversampled OFDM systems, in Proceedings International Conference on Digital Signal Processing (Santorini, Greece, July 1997)

11. C Siclet, P Siohan, D Pinchon, Perfect reconstruction conditions and design of oversampled DFT modulated transmultiplexers. Eurasip J. Appl. Signal Process, 14 pages (2006). Article ID 15756

12. G Cherubini, E Eleftheriou, S Ölçer, Filtered multitone modulation for very high-speed subscriber lines. IEEE J. Selected Areas Commun 20(5), 1016-1028 (2002)

13. H Lin, P Siohan, P Tanguy, JP Javaudin, An analysis of ElC for OFDM/OQAM systems. J. Commun. 4(1), 52-60 (2008)
14. G Ndo, H Lin, P Siohan, FBMC/OQAM equalization: exploiting the imaginary interference, in PIMRC'12 (Sydney, Australia, September 2012)

15. N Benvenuto, S Tomasin, L Tomba, Equalization methods in OFDM and FMT systems for broadband wireless communications. IEEE Trans. Commun. 50(9), 1413-1418 (2002)

16. R Zakaria, D Le Ruyet, On interference cancellation in Alamouti coding scheme for filter bank based multicarrier systems, in ISWCS (IImenau, Germany, June 2013)

17. G Fettweis, M Krondorf, S Bittner, GFDM-generalized frequency division multiplexing, in IEEE Vehicular Technology Conference (VTC Spring'09) (Barcelona, Spain, April 2009)

18. N Michailow, M Lentmaier, P Rost, G Fettweis, Integration of a GFDM secondary system in an OFDM primary system, in Future Network and Mobile Summit (FNMS'11) (Warsaw, January 2011)

19. R Datta, N Michailow, S Krone, M Lentmaier, G Fettweis, Generalized frequency division multiplexing in cognitive radio, in European Signal Processing Conference (EUSIPCO'12) (Bucharest, Romania, August 2012)

20. R Datta, N Michailow, M Lentmaier, G Fettweis, GFDM interference cancellation for flexible cognitive radio PHY design, in IEEE Vehicular Technology Conference (VTC Fall'12) (Quebec, Canada, September 2012)

21. R Datta, D Panaitopol, G Fettweis, Analysis of cyclostationary GFDM signal properties in flexible cognitive radio, in International Symposium on Communication and Information Technologies (ISCIT'12) (Gold Coast, Australia, October 2012)

22. N Michailow, I Gaspar, S Krone, M Lentmaier, G Fettweis, Generalized frequency division multiplexing: analysis of an alternative multi-carrier technique for next generation cellular systems, in International Symposium on Wireless Communiation System (ISWCS'12) (Paris, France, August 2012)

23. N Michailow, S Krone, M Lentmaier, G Fettweis, Bit error rate performance of generalized frequency division multiplexing, in IEEE Vehicular Technology Conference (VTC Fall'12) (Quebec, Canada, September 2012)

24. AM Tonello, A novel multi-carrier scheme: cyclic block filtered multitone modulation, in Proceedings of ICC 2013 (Budapest, June 2013)

25. HG Feichtinger, T Strohmer, Gabor Analysis and Algorithm - Theory and Applications, (Birkhäuser, Boston-Basel-Berlin, 1998)

26. B Le Floch, M Alard, C Berrou, Coded orthogonal frequency division multiplex. Proc. IEEE. 83, 982-996 (1995)

27. P Siohan, C Siclet, N Lacaille, Analysis and design of OFDM/OQAM systems based on filterbank theory. IEEE Trans. Signal Process. 50(5), 1170-1183 (2002)

28. PP Vaidyanathan, Multirate Systems and Filter Banks. (Prentice Hall, Englewood Cliffs, New York, New Jersey, 1993)

29. WY Zou, Y Wu, COFDM: an overview. IEEE Trans. Broadcast. 41(1), 1-8 (1995)

30. PD Welch, The use of fast Fourier transform for the estimation of power spectra: a method based on time averaging over short, modified periodograms. IEEE Trans. Audio Electroacoust. AU-15, 17-20 (1967)

31. FJ Harris, On the use of windows for harmonic analysis with the discrete Fourier transform. Proc. IEEE. 66(1), 51-83 (1978)

32. T Ihalainen, TH Stitz, M Rinne, M Renfors, Channel equalization in filter bank based multicarrier modulation for wireless communications. EURASIP J. Adv. Signal Process. 2007(ID 49389), 1-18 (2007)

33. T Ihalainen, A Ikhlef, J Louveaux, M Renfors, Channel equalization for multi-antenna FBMC/OQAM receivers. IEEE Trans. Vehic. Technol. 60(5), 2070-2085 (2011)

34. N Michailow, R Datta, S Krone, M Lentmaier, G Fettweis, Generalized frequency division multiplexing: a flexible multi-carrier modulation scheme for 5 th generation cellular networks, in Proceedings of the German Microwave Conference (GeMiC'12) (Ilmenau, Germany, March 2012)

35. JW Cooley, JW Tukey, An algorithm for machine computation of complex Fourier series. Math. Comput. 19(90), 297-301 (1965)

36. Y Dandach, P Siohan, FBMC/OQAM modulators with half complexity, in Proceedings Globecom'11 (Houston, December 2011)

37. H Lin, P Siohan, A new transceiver design for OFDM/OQAM modulation with cyclic prefix, in PIMRC'08 (Cannes, France, September 2008)

38. H Lin, Lélé C, P Siohan, A pseudo Alamouti transceiver design for OFDM/OQAM modulation with cyclic prefix, in SPAWC'09 (Perugia, Italy, June 2009), pp. 300-304 
39. M Renfors, T Ihalainen, TH Stitz, A block-Alamouti scheme for filter bank based multicarrier transmission, in Proceedings of the 2010 European Wireless Conference (Lucca, April 2010), pp. 1038-1041

40. R Zakaria, D Le Ruyet, A novel filter-bank multicarrier scheme to mitigate the intrinsic interference: application to MIMO systems. IEEE Trans. Wireless Commun. 11(3), 1112-1123 (2012)

41. MJ Abdoli, M Jia, J Ma, Weighted circularly convolved filtering in OFDM/OQAM, in PIMRC'13 (London, September 2013), pp. 8-11

doi:10.1186/1687-6180-2014-79

Cite this article as: Lin and Siohan: Multi-carrier modulation analysis and WCP-COQAM proposal. EURASIP Journal on Advances in Signal Processing 2014 2014:79.

Submit your manuscript to a SpringerOpen ${ }^{\odot}$ journal and benefit from:

- Convenient online submission

- Rigorous peer review

- Immediate publication on acceptance

- Open access: articles freely available online

- High visibility within the field

- Retaining the copyright to your article

Submit your next manuscript at $\gg$ springeropen.com 